\title{
Hybrid Effects of Carbon-Glass FRP Sheets in Combination with or without Concrete Beams
}

\author{
Thomas H.-K. Kang ${ }^{1), *}$, Woosuk Kim²), Sang-Su Ha ${ }^{3)}$, and Dong-Uk Choi ${ }^{4)}$
}

(Received September 2, 2013, Accepted November 18, 2013)

\begin{abstract}
The use of carbon fibers (CF) and glass fibers (GF) were combined to strengthen concrete flexural members. In this study, data of tensile tests of 94 hybrid carbon-glass FRP sheets and 47 carbon and GF rovings or sheets were thoroughly investigated in terms of tensile behavior. Based on comparisons between the rule of mixtures and test data, positive hybrid effects were identified for various $(\mathrm{GF} / \mathrm{CF})$ ratios. Unlike the rule of mixtures, the hybrid sheets with relatively low $(\mathrm{GF} / \mathrm{CF})$ ratios also produced pseudo-ductility. From the calibrated results obtained from experiments, a new analytical model for the stress-strain relationship of hybrid FRP sheets was proposed. Finally, the hybrid effects were verified by structural tests of concrete members strengthened with hybrid FRP sheets and either carbon or glass FRP sheets.
\end{abstract}

Keywords: carbon fibers, glass fibers, hybrid effects, fiber reinforced polymers, concrete, pseudo-ductility.

\section{Introduction}

Since the late 1980s fiber-reinforced polymer (FRP) sheets or wraps have been used to replace corrosion-vulnerable steel plates in repair applications. FRP sheets offer the advantages of light weight, high strength, low cost, constructability, and durability (non-corrosiveness). Despite the expensive cost relative to glass fibers (GF), carbon fibers (CF) and carbon FRP sheets/plates have been primarily used for repair and retrofit. This is mainly due to the fact that CF has a high elastic modulus and high ultimate strength (see Fig. 1). GF is popular, particularly for column jacketing (confining) retrofit, as it only costs about $5-10 \%$ as much as CF. GF has much less ultimate stress and very low elastic modulus (only about a quarter of that of steel), but very large ductility (Fig. 1). It is noted that aramid fibers (AF) have both very large ductility and relatively high elastic modulus (Fig. 1); however, because AF is as costly as CF, little economic advantage may be gained from the use of AF.

Brittleness is a major drawback of all these fibers (CF, AF and GF), since they have no yielding point and associated

\footnotetext{
${ }^{1)}$ Department of Architecture and Architectural Engineering, Seoul National University, Seoul 155-744, Korea.

*Corresponding Author; E-mail: tkang@snu.ac.kr

${ }^{2}$ Department of Architectural Engineering, Kumoh National Institute of Technology, Gumi 730-701, Korea.

${ }^{3)}$ Department of Architectural Engineering, Kangnam University, Yongin 446-702, Korea.

${ }^{4)}$ Department of Architectural Engineering, Hankyong National University, Anseong 456-749, Korea.
}

Copyright $($ The Author(s) 2014. This article is published with open access at Springerlink.com nonlinear behavior (Fig. 1). To improve the ductility of the fibers, a number of composite material science investigations have been conducted on hybrid fibrous composites (e.g., Bunsell and Harris 1974; Phillips 1976; Aveston and Sillwood 1976; Marom et al. 1978; Chou and Kelly 1980; Manders and Bader 1981; Miwa and Horiba 1994; Pan and Postle 1996). Applications of hybrid FRP composites, such as hybrid FRP bars and sheets combined with concrete, have been studied by several researchers (e.g., Nanni et al. 1994; Harris et al. 1998; Grace et al. 2002). The primary purpose of these civil engineering applications was to achieve "pseudo-ductility" similar to the ductile response of nonlinear steel materials. Pseudo-ductility can be defined in this paper as when, after the first fiber failure (first drop in load), the load carrying capacity is recovered or improved as the remaining fibers elongate. Pseudo-ductility is desirable because clear sound warning is produced during the first fiber failure, which indicates distress and the possible impending failure of structures.

The secondary purpose of hybrid FRP composites in civil engineering applications might be to actively utilize the socalled "hybrid effects." Marom et al. (1978) defined the hybrid effects as the deviation of the behavior of a hybrid composite from the rule of mixtures, while Manders and Bader (1981) simply defined it as the difference in behavior between a fiber in a hybrid composite and in a non-hybrid composite. Both positive and negative hybrid effects are possible; the effects are deemed positive when mechanical properties are above the prediction based on the rule of mixtures and vice versa for negative effects. It is extremely difficult to theoretically predict the hybrid effects and mechanical properties of hybrid fibrous composites, which are known to depend on the volumetric ratio of each fiber component, bonding property between the components, and 


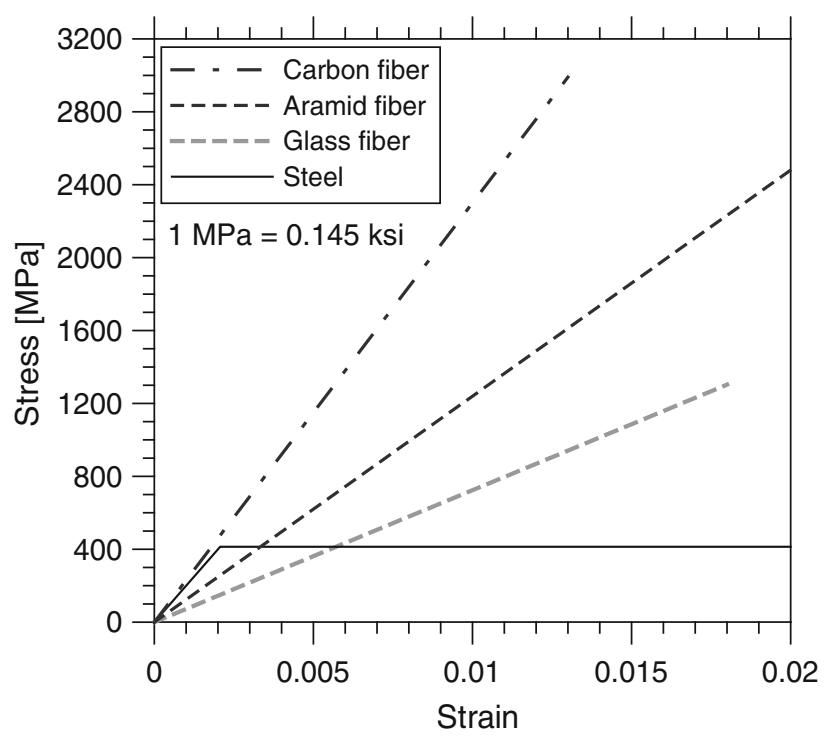

Fig. 1 Comparison of Young's moduli of steel and carbon, aramid and GF.

elastic moduli of the fibers or their ratio (Pan and Postle 1996). This is mainly due to the unavoidable uncertainty of the bonding property. Furthermore, the size effect is involved. In civil engineering applications, hybrid FRP sheets or plates consisting of fiber rovings (strands) would be practical and feasible. A high-strength CF roving is typically made of about 12,000 filaments $(12 \mathrm{~K})$ or multiples of 12,000 filaments (e.g., 24 or $48 \mathrm{~K}$ ), while an E-GF roving is made of 1,200, 2,200 tex or multiple of 2,200 tex, where 1 tex is $1,000 \mathrm{~m} / \mathrm{g}$. Figure 2 shows microscopic cross-sectional views of $\mathrm{CF}$ and GF rovings taken using a scanning electron microscopy of the University of Oklahoma. Thus, some findings from previous research on a micro-composite or a composite made of a fraction of different fibers embedded in the composite matrix (i.e., in the fiber roving or strand) may not be applicable to the hybrid FRP sheets that are focused on infrastructure repair or other civil engineering applications.

When the hybrid carbon-glass FRP sheet is subjected to tension, the CF with high elastic modulus and low ultimate strain ruptures first. The GF, with lower elastic modulus and higher ultimate strain, then takes over and resists the load. As noted, if the stress at GF rupture is equal to or higher than that at $\mathrm{CF}$ rupture, which depends on a volume ratio of $(\mathrm{GF} /$ CF) (e.g., Manders and Bader 1981), the pseudo-ductility can be obtained. Hybrid effects are additionally expected to be gained, such that it is possible to enhance (first) failure stress (or strain) beyond that predicted from the rule of mixtures, given Eq. (1) below:

$$
E_{H F}=E_{C F}\left(\frac{V_{C F}}{V_{H F}}\right)+E_{G F}\left(\frac{V_{G F}}{V_{H F}}\right)
$$

where $E_{H F}$ is the weighted mean elastic modulus of a carbon-glass hybrid composite; $E_{C F}$ and $E_{G F}$ are the elastic moduli of CF and GF, respectively; $V_{C F}$ and $V_{G F}$ are the CF and GF volumes, respectively; and $V_{H F}$ is the combined CF and GF volume or the volume of the hybrid composites.

Manders and Bader (1981) reported that the increase in strain at $\mathrm{CF}$ rupture in sandwich laminated hybrids would be about $50 \%$ of that of single CF, and Aveston and Sillwood (1976) also experimentally confirmed that the strain at CF rupture of hybrid carbon-glass-epoxy composites could be increased up to about 0.01. Furthermore, Miwa and Horiba (1994) suggested the empirical rule of "hybrid" mixtures as:

$$
f_{u \_C \_H F}=f_{u \_C F}\left(\frac{V_{C F}}{V_{H F}}\right)+f_{u \_G F}\left(\frac{V_{G F}}{V_{H F}}\right)
$$

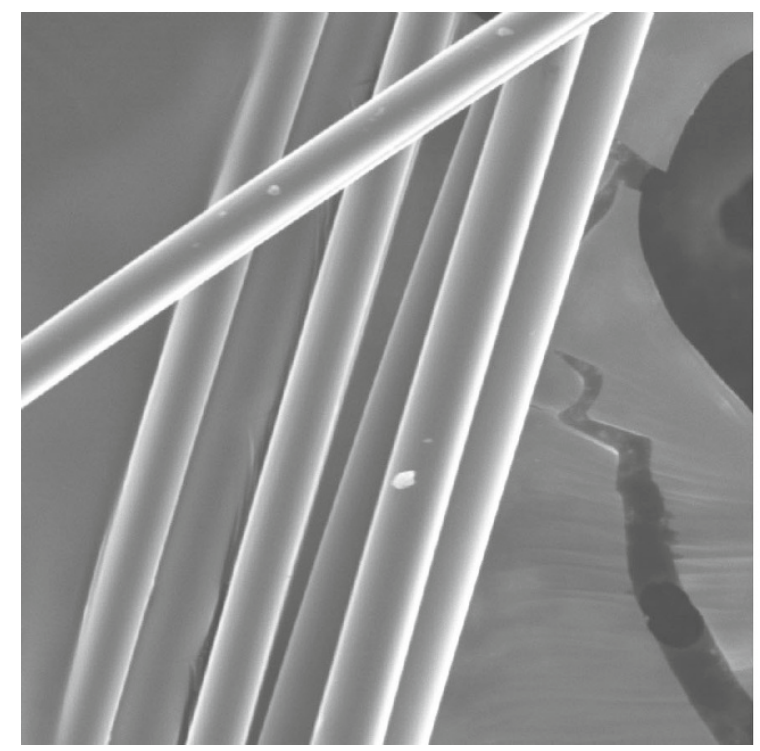

(a)

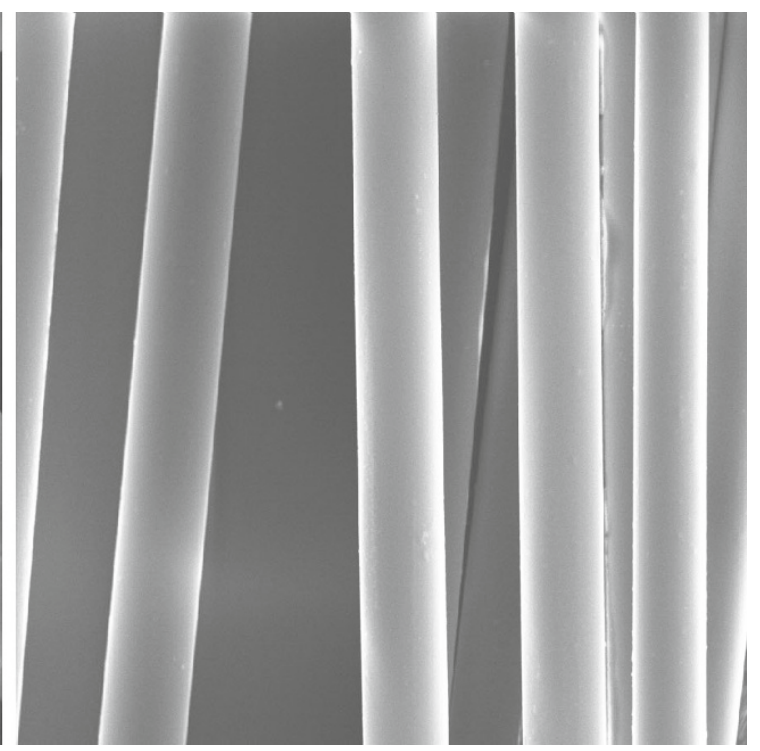

(b)

Fig. 2 Microscopic cross-sectional views of carbon and glass fibers taken by scanning electron microscopy. a CF ( $\times 1000)$, b GF $(\times 1000)$. 
where $f_{u_{-} C_{-} H F}$ is the mean stress of a carbon-glass hybrid composite at CF rupture and $f_{u_{-} C F}$ and $f_{u_{-} G F}$ are the ultimate stresses of CF and GF ruptures, respectively.

However, researchers (e.g., Manders and Bader 1981; Miwa and Horiba 1994) did not reach any definite conclusion on the ultimate stress of hybrid carbon-glass composites at GF rupture. Pan and Postle (1996) reported that due to the cross-coupling effects between the different fibers, a positive hybrid effect would be expected at the first fiber rupture, whereas a negative effect would be expected at the second fiber rupture; however, this appears to be the case only for the first fiber embedded in the matrix or a postulate without examination of an optimal ratio of two different fibers. It is not appropriate to apply the shear lag model (Cox 1952) to the case of interest, since the hybrid sheet may have a substantially different degree of interfacial shear stress as in the case of a short-fiber embedded in the matrix. The increased or decreased strain (or stress) at GF rupture of the hybrid composites, particularly hybrid FRP sheets that are common in civil engineering applications, have not been well studied. A continuous FRP sheet consisting of fiber rovings may have a moderate level of frictional coupling between GF and $\mathrm{CF}$ rovings and behave very differently than the microcomposites with a high level of frictional coupling.

This study consists of: (1) material test programs for identification of hybrid effects in the carbon-glass FRP sheets; (2) development of design models for stress-strain relationships with and without consideration of the hybrid effects; and (3) structural member-level verification of the hybrid effects.

\section{Material Test Programs}

Two independent material test programs on uniaxial hybrid FRP sheets (Fig. 3) and each corresponding fiber used for the fabrication of the hybrid sheets are presented in this section. Note that although ACI 440.2R-08, Section 4.3.1 recommends using mean minus three times standard deviations for the ultimate stress and strain from at least 20 samples, the number for the tested sample was $<20$ for all three test programs.

\subsection{First Test Program}

Rovings of high-strength CF and E-GF were tested in tension, hybrid carbon-glass FRP sheets in tension, and epoxy adhesives in bending ( $\mathrm{J}$ type). Both bare and impregnated rovings were tested (Fig. $4 \mathrm{a}, \mathrm{b}$ ), and digital data of forces and displacements at ultimate indicated by a Universal Test Machine (U.T.M.) with a $500 \mathrm{~N}$ capacity were manually recorded. The cross-sectional areas of CF and GF rovings were 0.886 and $0.444 \mathrm{~mm}^{2}$, respectively. This is based on each material's Specific Gravity $\left(\rho_{C F}=1.8\right.$;

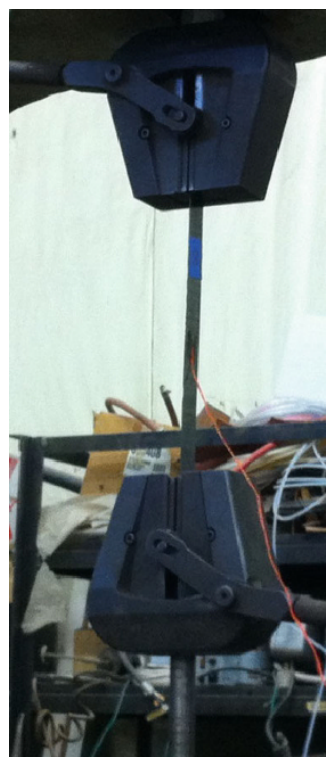

(a)

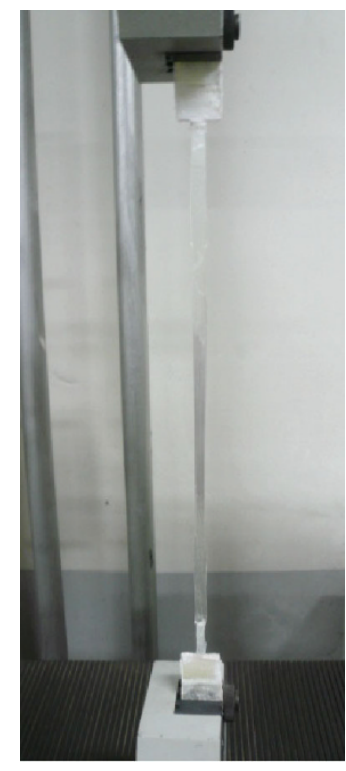

(b)
Fig. 4 Tensile tests of impregnated fiber rovings. a Tensile testing of impregnated CF roving, $\mathbf{b}$ tensile testing of impregnated GF roving.
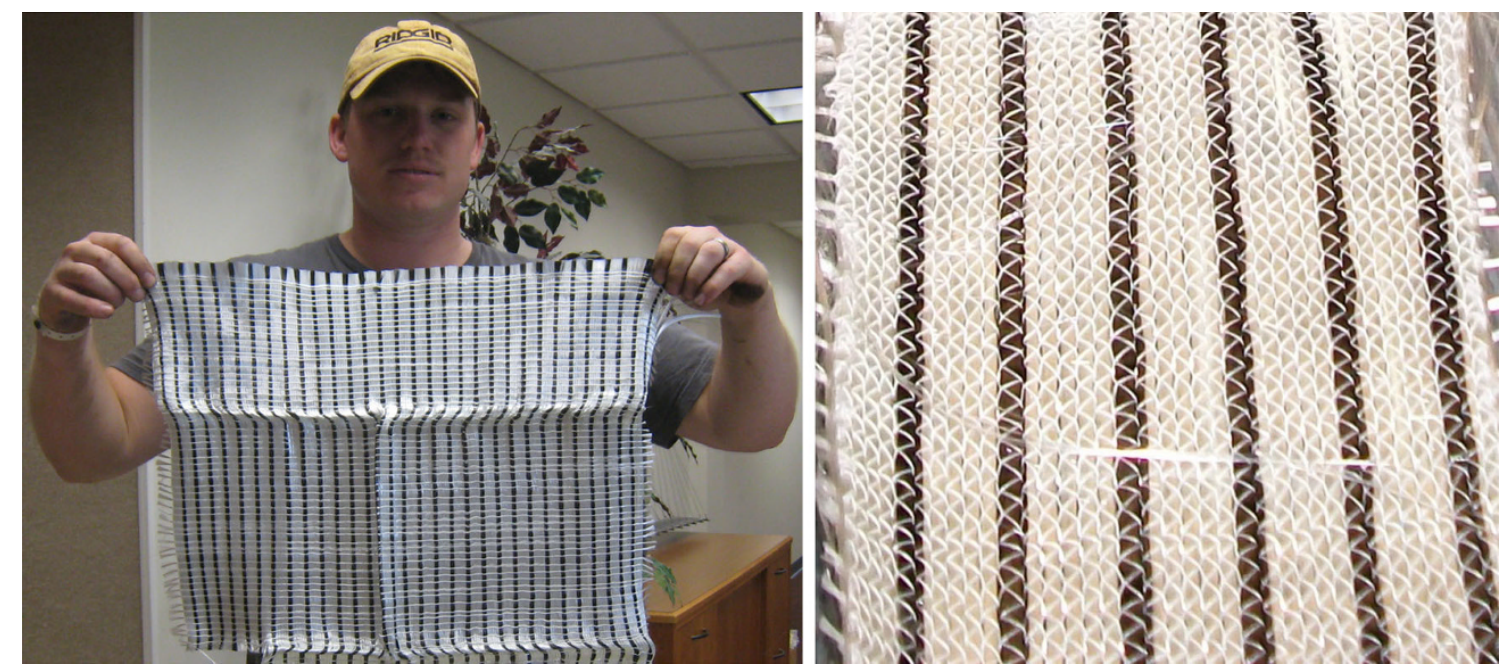

Fig. 3 Fabricated carbon-glass hybrid FRP sheets (black CF rovings, white GF rovings). 
$\rho_{G F}=2.54$ ), and the measured weight and length. The impregnated roving tests were carried out in accordance with ASTM D3039-08. The test specimens had a total length of $400 \mathrm{~mm}$ and an effective length between yarn grips of $260 \mathrm{~mm}$. The tensile loading speed was $10 \mathrm{~mm} / \mathrm{min}$.

For the bar roving tests, a pair of thin, flat plastic films were laminated in the grips. For the impregnated roving tests, two different types of grips (Types A and B) were developed. The Type A grip tabs were applied using an epoxy adhesive. According to ASTM D3039-08, no industry consensus on the grip at the end of the fiber coupon is available. Thus, although grip failures were not observed from any of the methods used in the first test program, an alternative method of gripping was developed, specifically, $90^{\circ}$ sandwich laminates using the same fiber rovings (Type $\mathrm{B}$ grip). Table 1 summarizes the average of the results from rovings categorized as non-impregnated, impregnated-Type A grip and impregnated-Type B grip. As shown, there were no significant discrepancies between the methods and within a method (standard deviations were relatively small), indicating that all of the test methods may yield consistent results.

Carbon-glass hybrid FRP sheets were fabricated with a (GF/CF) volumetric ratio of (8.8/1), and tested in tension in accordance with CSA S806-02. Table 2 indicates the averaged values for three samples of the hybrid FRP sheets. Each sample has a cross-sectional area of about $17.5 \mathrm{~mm}^{2}$, with a $48 \mathrm{~K}-\mathrm{CF}$ roving of $1.8 \mathrm{~mm}^{2}$ and eighteen 2,200 tex-GF rovings of $15.6 \mathrm{~mm}^{2}$. Both strain gauges and LVDTs embedded in the U.T.M. with a capacity of $1,200 \mathrm{kN}$ were used to digitally monitor stains or displacement. In this study, the LVDT data were more reliable than the strain gauge data.

In order to identify mechanical properties of epoxy adhesives, flexural tests were conducted instead of direct tensile tests. This is because, first, the flexural tests are much more convenient, and second, a tensile strain is generally smaller than the actual strain of the adhesive bonded to concrete (ASTM D638). In this study, epoxy solid blocks (J type) with dimensions of $25 \times 25 \times 240 \mathrm{~mm}$ were tested in flexure under four-point loading. The resulting average values of ultimate stress and strain and modulus of elasticity at rupture for three specimens are $42.8 \mathrm{MPa}, 0.0197$ and $2.19 \mathrm{GPa}$, respectively, which are similar to the typical values reported by ACI 503R-93. The ultimate strain of the product was relatively low. It would be useful to have an ultimate strain of about 0.04 to ensure that fiber ruptures prior to epoxy failure.

\subsection{Second Test Program}

Tensile tests of conventional carbon and glass FRP sheets and hybrid carbon-glass FRP sheets were conducted with a

Table 1 Measured results for the uniaxial tensile tests of CF and GF rovings (first test program) and provided properties of CF and GF filaments.

\begin{tabular}{|c|c|c|c|c|c|c|c|c|c|c|c|}
\hline & Carbon & & \multicolumn{9}{|c|}{ Glass filament } \\
\hline$\sigma_{u_{-} C F}$ & 4,900 & $\sigma_{u_{-} G F}$ & \multicolumn{9}{|c|}{2,900} \\
\hline$\varepsilon_{u_{-} C F}$ & 0.0213 & $\varepsilon_{u_{-} G F}$ & \multicolumn{9}{|c|}{0.0401} \\
\hline \multirow[t]{2}{*}{$E_{C F}$} & 230 & $E_{G F}$ & \multicolumn{9}{|c|}{72.4} \\
\hline & CF-N-1 & CF-N-2 & CF-N-3 & CF-N-4 & CF-N-5 & CF-N-6 & CF-N-7 & \multicolumn{4}{|c|}{ Average } \\
\hline$\sigma_{u_{-} C F}$ & 1,248 & 1,529 & 1,273 & 1,241 & 1,069 & 1,406 & 1,208 & \multicolumn{4}{|c|}{1,283} \\
\hline$\varepsilon_{u_{-} C F}$ & 0.011 & 0.0123 & 0.0117 & 0.0114 & 0.01 & 0.0125 & 0.0105 & \multicolumn{4}{|c|}{0.0113} \\
\hline \multirow[t]{2}{*}{$E_{C F}$} & 114 & 125 & 109 & 109 & 107 & 113 & 115 & \multicolumn{4}{|c|}{113} \\
\hline & CF-A-1 & CF-A-2 & CF-A-3 & CF-B-1 & CF-B-2 & CF-B-3 & CF-B-4 & CF-B-5 & CF-B-6 & & \\
\hline \multirow[t]{2}{*}{$\sigma_{u_{-} C F}$} & 1,734 & 1,680 & 1,382 & 1,628 & 1,544 & 1,354 & 1,515 & 1,533 & 1,624 & \multicolumn{2}{|c|}{1,553} \\
\hline & GF-N-1 & GF-N-2 & GF-N-3 & GF-N-4 & GF-N-5 & GF-N-6 & GF-N-7 & GF-N-8 & GF-N-9 & GF-N-10 & Average \\
\hline$\sigma_{u \_G F}$ & 708 & 883 & 757 & 776 & 901 & 769 & 801 & 794 & 785 & 742 & 792 \\
\hline$\varepsilon_{u \_G F}$ & 0.0167 & 0.0185 & 0.0177 & 0.0167 & 0.0209 & 0.0191 & 0.0174 & 0.0166 & 0.0154 & 0.0169 & 0.0176 \\
\hline \multirow[t]{2}{*}{$E_{G F}$} & 42.3 & 47.8 & 42.9 & 46.4 & 43.2 & 40.3 & 46.0 & 47.8 & 51.1 & 43.9 & 45.1 \\
\hline & GF-A-1 & GF-A-2 & GF-A-3 & GF-A-4 & GF-A-5 & GF-B-1 & GF-B-2 & GF-B-3 & GF-B-4 & GF-B-5 & Average \\
\hline$\sigma_{u_{-} G F}$ & 580 & 584 & 869 & 571 & 661 & 1052 & 951 & 890 & 799 & 681 & 764 \\
\hline
\end{tabular}

Filament properties are provided by the manufacturer.

$\sigma_{u_{-} C F}$ measured ultimate stress of CF roving (MPa); $\varepsilon_{u_{-} C F}$ measured ultimate strain of CF roving; $E_{C F}$ measured elastic modulus of CF roving $(\mathrm{GPa}) ; \sigma_{u_{G F}}$ measured ultimate stress of GF roving (MPa); $\varepsilon_{u_{-} G F}$ measured ultimate strain of GF roving; $E_{G F}$ Measured elastic modulus of GF roving $(\mathrm{GPa})$.

$C F$ carbon fiber roving, $G F$ glass fiber roving, $N$ non-impregnated rovings, $A$ impregnated-type A grip, $B$ impregnated-type B grip. 
Table 2 Measured and predicted results for the uniaxial tensile tests of hybrid FRP sheets with $(\mathrm{GF} / \mathrm{CF}=8.8 / 1)($ first test program).

\begin{tabular}{c|c|c|c|c|c|c|c|c|c|c|c}
\hline Specimen & $\begin{array}{c}\text { Meas. } \\
E_{H F}[\mathrm{GPa}]\end{array}$ & $\begin{array}{c}\text { Meas. } \\
\varepsilon_{u_{-} C_{-} H F}\end{array}$ & $\begin{array}{c}\text { Meas. } \\
\sigma_{u_{-} C_{-} H F} \\
(\mathrm{MPa})\end{array}$ & $E_{H F}^{\mathrm{a}}(\mathrm{GPa})$ & $\varepsilon_{u_{-} C_{-} H F}$ & $\begin{array}{c}\sigma_{u_{-} C_{-} H F} \\
(\mathrm{MPa})\end{array}$ & $\begin{array}{c}\sigma_{u_{-} C_{-} H F} \\
(\mathrm{MPa})\end{array}$ & $\begin{array}{c}\text { Meas. } E_{G F}^{*} \\
(\mathrm{GPa})\end{array}$ & $\begin{array}{c}\text { Meas. } \\
\varepsilon_{u_{-} G_{-} H F}\end{array}$ & $\begin{array}{c}\text { Meas. } \\
\sigma_{u_{-} G_{-} H F} \\
(\mathrm{MPa})\end{array}$ & $\begin{array}{c}\sigma_{u_{-} G}^{\mathrm{a}} H F \\
(\mathrm{MPa})\end{array}$ \\
\hline \hline Hybrid-1 & 59.2 & 0.0153 & 864.5 & 52 & 0.011 & 583.9 & 841.8 & 59 & 0.0218 & 1,040 & 711 \\
\hline Hybrid-2 & 67.1 & 0.0144 & 864.5 & 52 & 0.011 & 583.9 & 841.8 & 51.2 & 0.0181 & 928 & 711 \\
\hline Hybrid-3 & 68.5 & 0.0152 & 876.3 & 52 & 0.011 & 583.9 & 841.8 & 54 & 0.0228 & 1,277 & 711 \\
\hline Average & 64.9 & 0.0150 & 868.4 & 52 & 0.011 & 583.9 & 841.8 & 54.7 & 0.0209 & 1,082 & 711 \\
\hline
\end{tabular}

Meas. measured.

${ }^{\text {a }}$ Based on the rule of mixtures.

b Based on the rule of hybrid mixtures (Miwa and Horiba 1994).

$E_{H F}$ elastic modulus of hybrid FRP sheet; $\sigma_{u_{-} C_{-} H F}$ stress at CF rupture of hybrid FRP sheet; $\varepsilon_{u_{-} C_{-} H F}$ strain at CF rupture of hybrid FRP sheet; $E_{G F}^{*}$ average stress increase of hybrid FRP sheet divided by strain increase after CF rupture; $\sigma_{u_{-} G_{-} H F}$ stress at GF rupture of hybrid FRP sheet; $\varepsilon_{u_{-} G_{-} H F}$ strain at GF rupture of hybrid FRP sheet.

variety of $(\mathrm{GF} / \mathrm{CF})$ ratios ranging from (1/1) to (10/1). Each sheet had a total length of about $250 \mathrm{~mm}$. High-strength CF and E-GF rovings were used along with two different types of epoxy resins ( $\mathrm{J}$ type and $\mathrm{K}$ type) to fabricate a total of 99 impregnated hybrid sheet coupons. The J type epoxy is commonly used in practice, and the $\mathrm{K}$ type epoxy has a higher ductility. Tensile tests of the epoxy resins turned out to be infeasible due to the grip problem; thus, four-point loading tests were conducted on $25 \times 25 \times 240 \mathrm{~mm}$ molded epoxy blocks. The ultimate strains for the $\mathrm{J}$ and $\mathrm{K}$ types were measured to be 0.02 and 0.029 , respectively.

Using Specific Gravity $\left(\rho_{C F}=1.8\right.$ and $\left.\rho_{G F}=2.54\right)$ and measured weights and lengths, the cross-sectional areas of $\mathrm{CF}$ and GF rovings were calculated to be 0.45 and $0.455 \mathrm{~mm}^{2}$, respectively. Controlling the number of each fiber roving (12 K-CF roving and 1,200 tex-GF roving), hybrid FRP sheets with 12 different (GF/CF) ratios were made (Table 3), including carbon FRP and glass FRP sheets. However, all the data from the glass FRP sheets were misplaced. Each sheet was impregnated with epoxy resin in a mold, where overhead projector (OHP) films were used to make the sample detachable from the mold. The width of the sample ranged from 11.9 to $16.5 \mathrm{~mm}$, and the strip thickness was $1.5 \mathrm{~mm}$. An epoxy-to-fiber ratio of 1.5 was used.

Tension forces were digitally recorded from the U.T.M., tensile strains from the strain gauges mounted on the impregnated FRP sheet and total elongations from the LVDTs. The tensile loading speed was $1 \mathrm{~mm} / \mathrm{min}$.

\section{The Rule of Mixtures}

Chou and Kelly (1980) and Manders and Bader (1981) proposed a tensile stress model for hybrid carbon-glass FRP composites, as shown in Fig. 5, based on the rule of mixtures. Points $\mathrm{A}$ and $\mathrm{D}$ denote the ultimate tensile stresses when $\mathrm{GF}$ and $\mathrm{CF}$, respectively, are used alone (i.e., $\mathrm{GF}=100 \%$; $\mathrm{CF}=100 \%$ ). The lines A-E and B-D represent the mean stresses in hybrid FRP when GF and CF fail, respectively. The CF with lower $\varepsilon_{u_{-} C F}$ than $\varepsilon_{u_{-} G F}$ (or higher $E_{C F}$ than $E_{G F}$ ) always fails prior to GF. To the right of Point $\mathrm{C}$, after the first failure of $\mathrm{CF}$, the hybrid FRP has a very low residual mean stress that is only provided by GF (i.e., brittle failure). To the left of Point $\mathrm{C}$, even after the first failure of CF, the hybrid FRP with a relatively large amount of GF can sustain more loads without a drop in strength until the GF rupture. As such, pseudo-ductility can be achieved with this combination.

Points $\mathrm{A}$ and $\mathrm{D}$ are taken as $f_{u_{-} C F}$ and $f_{u_{-} G F}$, respectively, which can be obtained from the roving or sheet tests. The mean stress at Point $\mathrm{B}$ is calculated as $\varepsilon_{u_{-} C F}$ times $E_{G F}$, where $\varepsilon_{u} C F$ is the measured average ultimate strain of the $\mathrm{CF}$ roving/sheet and $E_{G F}$ is the measured elastic modulus of the GF roving/sheet. Using the rule of mixtures and the material properties, the $x$-axis value at Point $\mathrm{C}$ is determined (e.g., (4.4/1) for the first test program). The ratios of (4.4/1) and $(8.8 / 1)$ are equivalent to the carbon volume fractions of 18.4 and $10.2 \%$, respectively. The $y$-axis value can be obtained from a cross point of two straight lines drawn in Fig. $5 \mathrm{a}$ or $\mathrm{b}$.

To determine the stress $\left(f_{H F}\right)$ in hybrid FRP for a given tensile strain $\left(\varepsilon_{H F}\right)$, the rule of mixtures is applied as follows:

$$
\begin{aligned}
& \text { If } \varepsilon_{H F} \leq \varepsilon_{u_{-} C F} \\
& f_{H F}=\left[E_{C F}\left(\frac{A_{C F}}{A_{H F}}\right)+E_{G F}\left(\frac{A_{G F}}{A_{H F}}\right)\right] \varepsilon_{H F} \quad \text { or } \\
& f_{H F}=E_{H F} \varepsilon_{H F} \\
& \text { If } \quad \varepsilon_{u_{-} C F}<\varepsilon_{H F} \leq \varepsilon_{u_{-} G F} \times\left(A_{G F} / A_{H F}\right) \quad f_{H F}=E_{G F} \varepsilon_{H F}
\end{aligned}
$$

where $\varepsilon_{H F}$ and $f_{H F}$ are the strain and stress in hybrid FRP (variables), respectively; $\varepsilon_{u_{-} C F}$ and $\varepsilon_{u_{G} G F}$ are the ultimate strains of $\mathrm{CF}$ and $\mathrm{GF}$, respectively; $E_{C F}$ and $E_{G F}$ are the 
Table 3 Measured and predicted results for the uniaxial tensile tests (second test program).

\begin{tabular}{|c|c|c|c|c|c|c|c|c|c|c|c|}
\hline Specimen & $\begin{array}{c}\text { Meas. } E_{H F} \\
(\mathrm{GPa})\end{array}$ & $\begin{array}{c}\text { Meas. } \\
\varepsilon_{u_{-} C_{-} H F}\end{array}$ & $\begin{array}{c}\text { Meas. } \\
\sigma_{u_{-} C_{-} H F} \\
(\mathrm{MPa})\end{array}$ & $E_{H F}^{\mathrm{a}}(\mathrm{GPa})$ & $E_{u \_C \_H F}^{\mathrm{a}}$ & $\begin{array}{c}\sigma_{u_{-} C_{-} H F}^{\mathrm{a}} \\
(\mathrm{MPa})\end{array}$ & $\begin{array}{c}\sigma_{u_{-} C_{-} H F}^{\mathrm{b}}(\mathrm{MPa}) \\
(\mathrm{M})\end{array}$ & $\begin{array}{c}\text { Meas. } E_{G F}^{*} \\
(\mathrm{GPa})\end{array}$ & $\begin{array}{l}\text { Meas. } \\
\varepsilon_{u_{-} G \_H F}\end{array}$ & $\begin{array}{c}\text { Meas. } \\
\sigma_{u_{-} G_{-} H F} \\
(\mathrm{MPa})\end{array}$ & $\begin{array}{c}\sigma_{u_{-} G_{-} H F}^{\mathrm{a}}(\mathrm{MPa}) \\
(\mathrm{M} a\end{array}$ \\
\hline (1) & (2) & (3) & (4) & $(5)$ & $(6)$ & (7) & $(8)$ & (9) & $(10)$ & (11) & (12) \\
\hline CFRP-a & 273.8 & 0.01 & 2,425 & 202 & 0.0129 & 2,656 & 3,474 & & & & \\
\hline CFRP-b & 196 & 0.0125 & 2,450 & 202 & 0.0129 & 2,656 & 3,474 & & & & \\
\hline CFRP-c & 189.7 & 0.0146 & 2,597 & 202 & 0.0129 & 2,656 & 3,474 & & & & \\
\hline CFRP-d & 238.4 & 0.0105 & 2,496 & 202 & 0.0129 & 2,656 & 3,474 & & & & \\
\hline CFRP-e & 194 & 0.0145 & 2,660 & 202 & 0.0129 & 2,656 & 3,474 & & & & \\
\hline CFRP-D-a & 121.3 & 0.0108 & 2,404 & 202 & 0.0129 & 2,656 & 3,474 & & & & \\
\hline CFRP-D-b & 233.7 & 0.0153 & 3,555 & 202 & 0.0129 & 2,656 & 3,474 & & & & \\
\hline CFRP-D-c & 172.9 & 0.0154 & 2,662 & 202 & 0.0129 & 2,656 & 3,474 & & & & \\
\hline Average & 202.4 & 0.013 & 2,656 & 202 & 0.0129 & 2,656 & 3,474 & & & & \\
\hline HFRP-1-a & 130.9 & 0.0157 & 2,057 & 134.7 & 0.0129 & 1,738 & 1,920 & & & & 592 \\
\hline HFRP-1-b & 164.9 & 0.0125 & 1,815 & 134.7 & 0.0129 & 1,738 & 1,920 & & & & 592 \\
\hline HFRP-1-c & 145.3 & 0.0152 & 2,202 & 134.7 & 0.0129 & 1,738 & 1,920 & 244.3 & 0.0173 & 4,473 & 592 \\
\hline HFRP-1-d & 148 & 0.0179 & 2,644 & 134.7 & 0.0129 & 1,738 & 1,920 & & & & 592 \\
\hline HFRP-1-e & 156.5 & 0.0167 & 2,384 & 134.7 & 0.0129 & 1,738 & 1,920 & & & & 592 \\
\hline HFRP-1-f & 136.9 & 0.0142 & 1,945 & 134.7 & 0.0129 & 1,738 & 1,920 & & & & 592 \\
\hline HFRP-1-g & 130.9 & 0.0157 & 2,057 & 134.7 & 0.0129 & 1,738 & 1,920 & & & & 592 \\
\hline $\begin{array}{c}\text { HFRP-D1- } \\
\text { a }\end{array}$ & 150.5 & 0.0135 & 2,027 & 134.7 & 0.0129 & 1,738 & 1,920 & 208.9 & 0.0151 & 4,234 & 592 \\
\hline $\begin{array}{c}\text { HFRP-D1- } \\
\text { b }\end{array}$ & 144.6 & 0.0133 & 1,873 & 134.7 & 0.0129 & 1,738 & 1,920 & 238.7 & 0.016 & 4,030 & 592 \\
\hline $\begin{array}{c}\text { HFRP-D1- } \\
\text { c }\end{array}$ & 150.7 & 0.0134 & 2,077 & 134.7 & 0.0129 & 1,738 & 1,920 & & & & 592 \\
\hline Average & 146 & 0.0148 & 2,108 & 134.7 & 0.0129 & 1,738 & 1,920 & 230.6 & 0.0161 & 4,246 & 592 \\
\hline HFRP-2-a & 259.8 & 0.0053 & 1,477 & 112.2 & 0.0129 & 1,447 & 1,675 & & & & 790 \\
\hline HFRP-2-b & 99.4 & 0.0156 & 1,578 & 112.2 & 0.0129 & 1,447 & 1,675 & 143.8 & 0.0186 & 2,014 & 790 \\
\hline HFRP-2-c & 100.8 & 0.0135 & 1,357 & 112.2 & 0.0129 & 1,447 & 1,675 & 99.1 & 0.017 & 2,097 & 790 \\
\hline HFRP-2-d & 100.9 & 0.0132 & 1,328 & 112.2 & 0.0129 & 1,447 & 1,675 & & & & 790 \\
\hline HFRP-2-e & 92.8 & 0.0154 & 1,427 & 112.2 & 0.0129 & 1,447 & 1,675 & 126.8 & 0.0161 & 1,589 & 790 \\
\hline $\begin{array}{c}\text { HFRP-D2- } \\
\text { a }\end{array}$ & 126.7 & 0.0163 & 2,066 & 112.2 & 0.0129 & 1,447 & 1,675 & & & & 790 \\
\hline $\begin{array}{c}\text { HFRP-D2- } \\
\text { b }\end{array}$ & 121.3 & 0.0187 & 2,267 & 112.2 & 0.0129 & 1,447 & 1,675 & & & & 790 \\
\hline $\begin{array}{c}\text { HFRP-D2- } \\
\text { c }\end{array}$ & 106.5 & 0.018 & 1,756 & 112.2 & 0.0129 & 1,447 & 1,675 & & & & 790 \\
\hline Average & 126 & 0.0145 & 1,657 & 112.2 & 0.0129 & 1,447 & 1,675 & 123.2 & 0.0172 & 1,900 & 790 \\
\hline HFRP-3-a & 106.3 & 0.0191 & 2,039 & 101 & 0.0129 & 1,303 & 1,552 & & & & 888 \\
\hline HFRP-3-b & 89.1 & 0.0095 & 847 & 101 & 0.0129 & 1,303 & 1,552 & & & & 888 \\
\hline HFRP-3-c & 114.7 & 0.0135 & 1,553 & 101 & 0.0129 & 1,303 & 1,552 & & & & 888 \\
\hline HFRP-3-d & 117.5 & 0.013 & 1,527 & 101 & 0.0129 & 1,303 & 1,552 & 65.5 & & & 888 \\
\hline HFRP-3-e & 111.8 & 0.0168 & 1,883 & 101 & 0.0129 & 1,303 & 1,552 & 111.8 & 0.0195 & 2,447 & 888 \\
\hline
\end{tabular}


Table 3 continued.

\begin{tabular}{|c|c|c|c|c|c|c|c|c|c|c|c|}
\hline Specimen & $\begin{array}{c}\text { Meas. } E_{H F} \\
(\mathrm{GPa})\end{array}$ & $\begin{array}{l}\text { Meas. } \\
\varepsilon_{u_{-} C_{-} H F}\end{array}$ & $\begin{array}{c}\text { Meas. } \\
\sigma_{u_{-} C_{-} H F} \\
(\mathrm{MPa})\end{array}$ & $E_{H F}^{\mathrm{a}}(\mathrm{GPa})$ & $E_{u_{-} C_{-} H F}^{\mathrm{a}}$ & $\begin{array}{c}\sigma_{u_{-} C_{-} H F}^{\mathrm{a}} \\
(\mathrm{MPa})\end{array}$ & $\begin{array}{c}\sigma_{u_{-} C_{-} H F}^{\mathrm{b}} \\
(\mathrm{MPa})\end{array}$ & $\begin{array}{c}\text { Meas. } E_{G F}^{*} \\
(\mathrm{GPa})\end{array}$ & $\begin{array}{c}\text { Meas. } \\
\varepsilon_{u \_G \_H F}\end{array}$ & $\begin{array}{c}\text { Meas. } \\
\sigma_{u_{-} G \_H F} H \\
(\mathrm{MPa})\end{array}$ & $\begin{array}{c}\sigma_{u_{-} G_{-} H F}^{\mathrm{a}}(\mathrm{MPa}) \\
(\mathrm{M} a\end{array}$ \\
\hline (1) & (2) & (3) & (4) & (5) & (6) & (7) & (8) & (9) & (10) & $(11)$ & (12) \\
\hline HFRP-3-f & 91.6 & 0.0166 & 1,488 & 101 & 0.0129 & 1,303 & 1,552 & & & & 888 \\
\hline HFRP-3-g & 97.4 & 0.0124 & 1,203 & 101 & 0.0129 & 1,303 & 1,552 & & & & 888 \\
\hline $\begin{array}{c}\text { HFRP-D3- } \\
\text { a }\end{array}$ & 109.4 & 0.0171 & 1,875 & 101 & 0.0129 & 1,303 & 1,552 & & & & 888 \\
\hline $\begin{array}{c}\text { HFRP-D3- } \\
\text { b }\end{array}$ & 92.9 & 0.0164 & 1,590 & 101 & 0.0129 & 1,303 & 1,552 & & & & 888 \\
\hline $\begin{array}{c}\text { HFRP-D3- } \\
\text { c }\end{array}$ & 104.1 & 0.0184 & 1,914 & 101 & 0.0129 & 1,303 & 1,552 & & & & 888 \\
\hline Average & 103.5 & 0.0153 & 1,592 & 101 & 0.0129 & 1,303 & 1,552 & 88.7 & 0.0195 & 2,447 & 888 \\
\hline HFRP-4-a & 107.5 & 0.0178 & 1,968 & 94.2 & 0.0129 & 1,215 & 1,479 & & & & 948 \\
\hline HFRP-4-b & 93.6 & 0.0185 & 1,729 & 94.2 & 0.0129 & 1,215 & 1,479 & & & & 948 \\
\hline HFRP-4-c & 88.3 & 0.021 & 1,854 & 94.2 & 0.0129 & 1,215 & 1,479 & & & & 948 \\
\hline HFRP-4-d & 101.6 & 0.0191 & 1,939 & 94.2 & 0.0129 & 1,215 & 1,479 & & & & 948 \\
\hline HFRP-4-e & 108.7 & 0.016 & 1,725 & 94.2 & 0.0129 & 1,215 & 1,479 & & & & 948 \\
\hline HFRP-4-f & 87.3 & 0.0193 & 1,684 & 94.2 & 0.0129 & 1,215 & 1,479 & & & & 948 \\
\hline HFRP-4-g & 96.9 & 0.0183 & 1,694 & 94.2 & 0.0129 & 1,215 & 1,479 & & & & 948 \\
\hline $\begin{array}{c}\text { HFRP-D4- } \\
\text { a }\end{array}$ & 98.7 & 0.0178 & 1,757 & 94.2 & 0.0129 & 1,215 & 1,479 & & & & 948 \\
\hline $\begin{array}{c}\text { HFRP-D4- } \\
\text { b }\end{array}$ & 104.5 & 0.0185 & 1,936 & 94.2 & 0.0129 & 1,215 & 1,479 & & & & 948 \\
\hline $\begin{array}{c}\text { HFRP-D4- } \\
\text { c }\end{array}$ & 103.6 & 0.0172 & 1,889 & 94.2 & 0.0129 & 1,215 & 1,479 & & & & 948 \\
\hline Average & 99.1 & 0.0184 & 1,818 & 94.2 & 0.0129 & 1,215 & 1,479 & & & & 948 \\
\hline HFRP-5-a & 77.9 & 0.0161 & 1,253 & 89.4 & 0.0129 & 1,153 & 1,426 & & & & 990 \\
\hline HFRP-5-b & 79.1 & 0.0197 & 1,556 & 89.4 & 0.0129 & 1,153 & 1,426 & & & & 990 \\
\hline HFRP-5-c & 31.9 & 0.0174 & 554 & 89.4 & 0.0129 & 1,153 & 1,426 & & & & 990 \\
\hline HFRP-5-d & 78.2 & 0.0178 & 1,362 & 89.4 & 0.0129 & 1,153 & 1,426 & & & & 990 \\
\hline HFRP-5-e & 80.3 & 0.0201 & 1,618 & 89.4 & 0.0129 & 1,153 & 1,426 & & & & 990 \\
\hline $\begin{array}{c}\text { HFRP-D5- } \\
\text { a }\end{array}$ & 69.8 & 0.0192 & 1,834 & 89.4 & 0.0129 & 1,153 & 1,426 & & & & 990 \\
\hline $\begin{array}{c}\text { HFRP-D5- } \\
\text { b }\end{array}$ & 97.2 & 0.018 & 1,753 & 89.4 & 0.0129 & 1,153 & 1,426 & & & & 990 \\
\hline $\begin{array}{c}\text { HFRP-D5- } \\
\text { c }\end{array}$ & 99 & 0.0185 & 1,834 & 89.4 & 0.0129 & 1,153 & 1,426 & & & & 990 \\
\hline Average & 76.7 & 0.0184 & 1,471 & 89.4 & 0.0129 & 1,153 & 1,426 & & & & 990 \\
\hline HFRP-6-a & 74.4 & 0.0185 & 1,375 & 86.3 & 0.0129 & 1,113 & 1,392 & 71.2 & & & 1,018 \\
\hline HFRP-6-b & 80.9 & 0.0175 & 1,417 & 86.3 & 0.0129 & 1,113 & 1,392 & & & & 1,018 \\
\hline HFRP-6-c & 80.9 & 0.0177 & 1,430 & 86.3 & 0.0129 & 1,113 & 1,392 & & & & 1,018 \\
\hline HFRP-6-d & 80.6 & 0.018 & 1,450 & 86.3 & 0.0129 & 1,113 & 1,392 & 84.1 & 0.0193 & 1,726 & 1,018 \\
\hline HFRP-6-e & 78.5 & 0.0199 & 1,559 & 86.3 & 0.0129 & 1,113 & 1,392 & & & & 1,018 \\
\hline HFRP-6-f & 81.2 & 0.0193 & 1,565 & 86.3 & 0.0129 & 1,113 & 1,392 & & & & 1,018 \\
\hline
\end{tabular}


Table 3 continued.

\begin{tabular}{|c|c|c|c|c|c|c|c|c|c|c|c|}
\hline Specimen & $\begin{array}{c}\text { Meas. } E_{H F} \\
(\mathrm{GPa})\end{array}$ & $\begin{array}{l}\text { Meas. } \\
\varepsilon_{u_{-} C_{-} H F}\end{array}$ & $\begin{array}{c}\text { Meas. } \\
\sigma_{u_{-} C_{-} H F} \\
(\mathrm{MPa})\end{array}$ & $E_{H F}^{\mathrm{a}}(\mathrm{GPa})$ & $E_{u_{-} C_{-} H F}^{\mathrm{a}}$ & $\begin{array}{c}\sigma_{u_{-} C_{-} H F}^{\mathrm{a}} \\
(\mathrm{MPa})\end{array}$ & $\begin{array}{c}\sigma_{u_{-} C_{-} H F}^{\mathrm{b}}(\mathrm{MPa}) \\
\left(\mathrm{M}-{ }^{2}\right.\end{array}$ & $\begin{array}{c}\text { Meas. } E_{G F}^{*} \\
(\mathrm{GPa})\end{array}$ & $\begin{array}{c}\text { Meas. } \\
\varepsilon_{u_{-} G \_H F}\end{array}$ & $\begin{array}{c}\text { Meas. } \\
\sigma_{u_{-} G_{-} H F} \\
(\mathrm{MPa})\end{array}$ & $\begin{array}{c}\sigma_{u_{-} G_{-} H F}^{\mathrm{a}}(\mathrm{MPa}) \\
(\mathrm{M} a\end{array}$ \\
\hline (1) & (2) & (3) & (4) & (5) & (6) & (7) & (8) & (9) & (10) & $(11)$ & (12) \\
\hline $\begin{array}{c}\text { HFRP-D6- } \\
\mathrm{a}\end{array}$ & 82.3 & 0.0178 & 1,465 & 86.3 & 0.0129 & 1,113 & 1,392 & 53.6 & 0.0233 & 1,511 & 1,018 \\
\hline $\begin{array}{c}\text { HFRP-D6- } \\
\text { b }\end{array}$ & 92.6 & 0.0207 & 1,783 & 86.3 & 0.0129 & 1,113 & 1,392 & & & & 1,018 \\
\hline $\begin{array}{c}\text { HFRP-D6- } \\
\text { c }\end{array}$ & 93.8 & 0.0188 & 1,767 & 86.3 & 0.0129 & 1,113 & 1,392 & & & & 1,018 \\
\hline Average & 82.8 & 0.0187 & 1,535 & 86.3 & 0.0129 & 1,113 & 1,392 & 69.6 & 0.0213 & 1,619 & 1,018 \\
\hline HFRP-7-a & 68.2 & 0.0193 & 1,313 & 83.9 & 0.0129 & 1,082 & 1,366 & & & & 1,038 \\
\hline HFRP-7-b & 73.6 & 0.0173 & 1,269 & 83.9 & 0.0129 & 1,082 & 1,366 & & & & 1,038 \\
\hline HFRP-7-c & 61.9 & 0.0166 & 1,027 & 83.9 & 0.0129 & 1,082 & 1,366 & & & & 1,038 \\
\hline HFRP-7-d & 78.6 & 0.0158 & 1,248 & 83.9 & 0.0129 & 1,082 & 1,366 & & & & 1,038 \\
\hline HFRP-7-e & 66.8 & 0.0178 & 1,192 & 83.9 & 0.0129 & 1,082 & 1,366 & & & & 1,038 \\
\hline HFRP-7-f & 68 & 0.021 & 1,604 & 83.9 & 0.0129 & 1,082 & 1,366 & & & & 1,038 \\
\hline HFRP-7-g & 81.6 & 0.0176 & 1,464 & 83.9 & 0.0129 & 1,082 & 1,366 & 62.3 & 0.02 & 1,486 & 1,038 \\
\hline $\begin{array}{c}\text { HFRP-D7- } \\
\text { a }\end{array}$ & 87.3 & 0.0193 & 1,682 & 83.9 & 0.0129 & 1,082 & 1,366 & 118.3 & 0.0229 & 1,918 & 1,038 \\
\hline $\begin{array}{c}\text { HFRP-D7- } \\
\text { b }\end{array}$ & 91.6 & 0.0203 & 1,863 & 83.9 & 0.0129 & 1,082 & 1,366 & & & & 1,038 \\
\hline $\begin{array}{c}\text { HFRP-D7- } \\
\text { c }\end{array}$ & 79.7 & 0.0205 & 1,645 & 83.9 & 0.0129 & 1,082 & 1,366 & & & & 1,038 \\
\hline Average & 75.7 & 0.0186 & 1,431 & 83.9 & 0.0129 & 1,082 & 1,366 & 90.3 & 0.0215 & 1,702 & 1,038 \\
\hline HFRP-8-a & 71.4 & 0.018 & 1,286 & 82.1 & 0.0129 & 1,059 & 1,346 & & & & 1,054 \\
\hline HFRP-8-b & 73.1 & 0.0211 & 1,543 & 82.1 & 0.0129 & 1,059 & 1,346 & & & & 1,054 \\
\hline HFRP-8-c & 71.3 & 0.0173 & 1,236 & 82.1 & 0.0129 & 1,059 & 1,346 & & & & 1,054 \\
\hline HFRP-8-d & 74.9 & 0.02 & 1,454 & 82.1 & 0.0129 & 1,059 & 1,346 & 40.1 & & & 1,054 \\
\hline HFRP-8-e & 73.7 & 0.0184 & 1,356 & 82.1 & 0.0129 & 1,059 & 1,346 & 58.2 & 0.0233 & 1,359 & 1,054 \\
\hline HFRP-8-f & 88 & 0.0206 & 1,811 & 82.1 & 0.0129 & 1,059 & 1,346 & 56 & 0.0251 & 1,772 & 1,054 \\
\hline HFRP-8-g & 79.8 & 0.0221 & 1,766 & 82.1 & 0.0129 & 1,059 & 1,346 & 49 & & & 1,054 \\
\hline $\begin{array}{c}\text { HFRP-D8- } \\
\mathrm{a}\end{array}$ & 85.4 & 0.0179 & 1,529 & 82.1 & 0.0129 & 1,059 & 1,346 & & & & 1,054 \\
\hline $\begin{array}{c}\text { HFRP-D8- } \\
\mathrm{b}\end{array}$ & 82.2 & 0.0184 & 1,514 & 82.1 & 0.0129 & 1,059 & 1,346 & & & & 1,054 \\
\hline $\begin{array}{c}\text { HFRP-D8- } \\
\text { c }\end{array}$ & 89.8 & 0.0141 & 1,263 & 82.1 & 0.0129 & 1,059 & 1,346 & & & & 1,054 \\
\hline Average & 79 & 0.0188 & 1,476 & 82.1 & 0.0129 & 1,059 & 1,346 & 50.8 & 0.0242 & 1,566 & 1,054 \\
\hline HFRP-9-a & 84.2 & 0.0211 & 1,777 & 80.6 & 0.0129 & 1,040 & 1,330 & 92.8 & 0.0245 & 1,766 & 1,067 \\
\hline HFRP-9-b & 87.2 & 0.0211 & 1,837 & 80.6 & 0.0129 & 1,040 & 1,330 & 107.2 & 0.0235 & 1,833 & 1,067 \\
\hline HFRP-9-c & 89.1 & 0.0203 & 1,807 & 80.6 & 0.0129 & 1,040 & 1,330 & 79.5 & 0.0251 & 1,841 & 1,067 \\
\hline HFRP-9-d & 101.8 & 0.0193 & 1,697 & 80.6 & 0.0129 & 1,040 & 1,330 & & & & 1,067 \\
\hline HFRP-9-e & 99.4 & 0.0171 & 1,717 & 80.6 & 0.0129 & 1,040 & 1,330 & 53.8 & 0.0233 & 1,865 & 1,067 \\
\hline HFRP-9-f & 94.5 & 0.0192 & 1,986 & 80.6 & 0.0129 & 1,040 & 1,330 & 67.2 & & & 1,067 \\
\hline
\end{tabular}


Table 3 continued.

\begin{tabular}{|c|c|c|c|c|c|c|c|c|c|c|c|}
\hline Specimen & $\begin{array}{c}\text { Meas. } E_{H F} \\
(\mathrm{GPa})\end{array}$ & $\begin{array}{l}\text { Meas. } \\
\varepsilon_{u_{-} C_{-} H F}\end{array}$ & $\begin{array}{c}\text { Meas. } \\
\sigma_{u_{-} C_{-} H F} \\
(\mathrm{MPa})\end{array}$ & $E_{H F}^{\mathrm{a}}(\mathrm{GPa})$ & $E_{u_{-} C_{-} H F}^{\mathrm{a}}$ & $\begin{array}{c}\sigma_{u_{-} C_{-} H F}^{\mathrm{a}} \\
(\mathrm{MPa})\end{array}$ & $\begin{array}{c}\sigma_{u_{-} C_{-} H F}^{\mathrm{b}}(\mathrm{MPa}) \\
\left(\mathrm{M}-{ }^{2}\right.\end{array}$ & $\begin{array}{c}\text { Meas. } E_{G F}^{*} \\
(\mathrm{GPa})\end{array}$ & $\begin{array}{c}\text { Meas. } \\
\varepsilon_{u_{-} G_{-} H F}\end{array}$ & $\begin{array}{c}\text { Meas. } \\
\sigma_{u_{-} G \_H F} H \\
(\mathrm{MPa})\end{array}$ & $\begin{array}{c}\sigma_{u_{-} G_{H} H F}^{\mathrm{a}} \\
(\mathrm{MPa})\end{array}$ \\
\hline (1) & (2) & (3) & (4) & (5) & (6) & (7) & (8) & (9) & (10) & $(11)$ & (12) \\
\hline HFRP-9-g & 78.5 & 0.0201 & 1,580 & 80.6 & 0.0129 & 1,040 & 1,330 & & & & 1,067 \\
\hline $\begin{array}{c}\text { HFRP-D9- } \\
\mathrm{a}\end{array}$ & 84.4 & 0.0187 & 1,645 & 80.6 & 0.0129 & 1,040 & 1,330 & 36.2 & 0.0192 & 1,715 & 1,067 \\
\hline $\begin{array}{c}\text { HFRP-D9- } \\
\mathrm{b}\end{array}$ & 92.3 & 0.0162 & 1,149 & 80.6 & 0.0129 & 1,040 & 1,330 & 20.6 & 0.0222 & 1,560 & 1,067 \\
\hline $\begin{array}{c}\text { HFRP-D9- } \\
\text { c }\end{array}$ & 82.9 & 0.0223 & 1,548 & 80.6 & 0.0129 & 1,040 & 1,330 & & & & 1,067 \\
\hline Average & 89.4 & 0.0195 & 1,674 & 80.6 & 0.0129 & 1,040 & 1,330 & 65.3 & 0.023 & 1,763 & 1,067 \\
\hline HFRP-10-a & 73.8 & 0.0209 & 1,544 & 79.4 & 0.0129 & 1,024 & 1,317 & & & & 1,078 \\
\hline HFRP-10-b & 74.7 & 0.021 & 1,568 & 79.4 & 0.0129 & 1,024 & 1,317 & 100.8 & & & 1,078 \\
\hline HFRP-10-c & 70.2 & 0.0205 & 1,439 & 79.4 & 0.0129 & 1,024 & 1,317 & & & & 1,078 \\
\hline $\begin{array}{l}\text { HFRP- } \\
\text { D10-a }\end{array}$ & 86.5 & 0.0187 & 1,621 & 79.4 & 0.0129 & 1,024 & 1,317 & & & & 1,078 \\
\hline $\begin{array}{l}\text { HFRP- } \\
\text { D10-b }\end{array}$ & 85 & 0.0171 & 1,396 & 79.4 & 0.0129 & 1,024 & 1,317 & 66.7 & 0.0205 & 1,494 & 1,078 \\
\hline $\begin{array}{l}\text { HFRP- } \\
\text { D10-c }\end{array}$ & 88.3 & 0.0143 & 1,305 & 79.4 & 0.0129 & 1,024 & 1,317 & & & & 1,078 \\
\hline Average & 79.8 & 0.0188 & 1,479 & 79.4 & 0.0129 & 1,024 & 1,317 & 83.8 & 0.0205 & 1,494 & 1,078 \\
\hline
\end{tabular}

$1 \rightarrow(\mathrm{GF} / \mathrm{CF}=1 / 1) ; 2 \rightarrow(\mathrm{GF} / \mathrm{CF}=2 / 1) ; 3 \rightarrow(\mathrm{GF} / \mathrm{CF}=3 / 1) ; 4 \rightarrow(\mathrm{GF} / \mathrm{CF}=4 / 1) ; 5 \rightarrow(\mathrm{GF} / \mathrm{CF}=5.1 / 1) ; 6 \rightarrow(\mathrm{GF} / \mathrm{CF}=6.1 / 1) ;$

$7 \rightarrow(\mathrm{GF} / \mathrm{CF}=7.1 / 1) ; 8 \rightarrow(\mathrm{GF} / \mathrm{CF}=8.1 / 1) ; 9 \rightarrow(\mathrm{GF} / \mathrm{CF}=9.1 / 1) ; 10 \rightarrow(\mathrm{GF} / \mathrm{CF}=10.1 / 1)$.

HFRP hybrid FRP sheet, $D$ ductile K type epoxy.

$E_{H F}$ elastic modulus of hybrid FRP sheet; $\sigma_{u_{-} C_{-} H F}$ stress at CF rupture of hybrid FRP sheet; $\varepsilon_{u_{-} C_{-} H F}$ strain at CF rupture of hybrid FRP sheet; $E_{G F}^{*}$ average increase in stress of hybrid FRP sheet after CF rupture divided by increase in strain; $\sigma_{u_{-} G_{-} H F}$ stress at GF rupture of hybrid FRP sheet; $\varepsilon_{u_{-} G \_H F}$ strain at GF rupture of hybrid FRP sheet.

${ }^{\text {a }}$ Based on the rule of mixtures.

${ }^{b}$ Based on the rule of hybrid mixtures (Miwa and Horiba 1994).
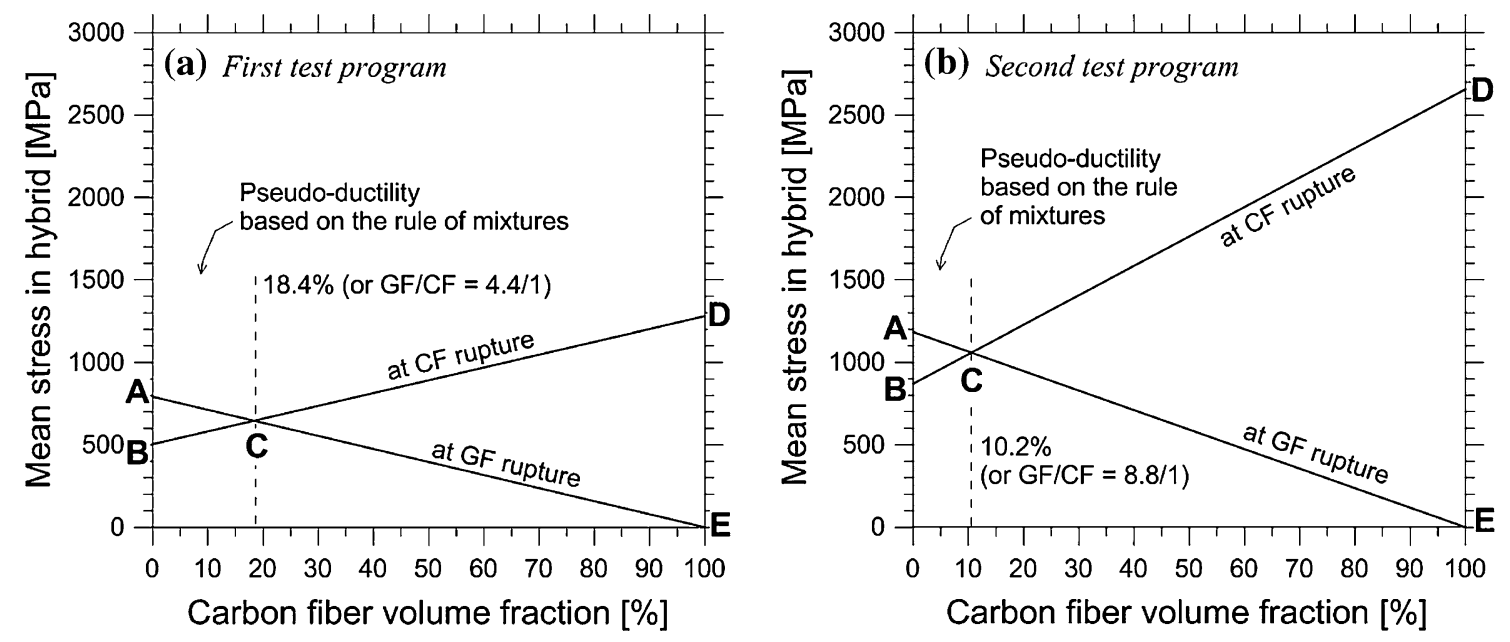

Fig. 5 The rule of mixtures.

elastic moduli of CF and GF, respectively; $A_{C F}$ and $A_{G F}$ are the cross-sectional areas of $\mathrm{CF}$ and GF in a hybrid sheet coupon, and $A_{H F}$ is $\left(A_{C F}+A_{G F}\right)$.
Equations (3) and (4) are consistent with Fig. 5. For example, when the fiber roving properties of the first test program are considered and the carbon volume fraction is 
$18.4 \%$, the stress of $f_{H F}$ is calculated to be $\left[\left(0.184 E_{C F}+\right.\right.$ $\left.\left.0.816 E_{G F}\right) \times \varepsilon_{H F}\right]$ at $\mathrm{CF}$ rupture, which is represented by Point $\mathrm{C}$ in Fig. 5a. Once CF rupture occurs Eq. (4) is applicable and the stress at GF rupture is assumed to be the same as $\left[\varepsilon_{u_{-} G F} E_{G F} \times\left(A_{G F} / A_{H F}\right)\right]$. This failure is represented by the line $\mathrm{A}-\mathrm{E}$ in Fig. 5 .

\section{Assessment of Test Results}

Based on the information relevant to the mechanical properties of materials obtained in the test programs, an attempt was made to identify two different hybrid effects: (1) improved mechanical properties until the first peak, and (2) those after the first peak. Assessment of the data was carefully undertaken in this section.

\subsection{First Test Program}

First, the roving tensile properties were obtained (Table 1). Using each fiber's Specific Gravity $\left(\rho_{C F}=1.8 ; \rho_{G F}=2.54\right)$ and measured weight per unit length, the cross-sectional area was determined to be 0.444 and $0.866 \mathrm{~mm}^{2}$ for the tested $\mathrm{CF}$ and GF rovings, respectively. The weight of the fiber roving was measured using a digital scale with an accuracy of $\pm 0.01 \mathrm{~g}$.

Results from the tensile tests are presented in Table 1. In the remainder of the paper, the mean stress and strain $\left(f_{u}\right.$ and $\left.\varepsilon_{u}\right)$ from the non-impregnated (bare) coupon tests were used (though both results are similar). This is because epoxy resins are typically applied on only one side of the FRP sheet. The roving results for ultimate stress $\left(f_{u}\right)$, ultimate strain $\left(\varepsilon_{u}\right)$ and elastic modulus $(E)$ in tension are substantially lower than the filament properties provided by the manufacturer (Table 1). This is due to the fragmentation process that generates unequal tension of filaments within a roving and failure strain variation between the filaments. Such phenomena are also seen from the behavior of conventional FRP sheets externally bonded to the concrete surface.

As indicated in Table 2 [compare Columns (4) and (7), and (11) and (12)], both stresses at CF and GF ruptures in hybrid FRP sheets with a $(\mathrm{GF} / \mathrm{CF})$ ratio of $(8.8 / 1)$ were higher than the stress values of $\mathrm{CF}$ and GF rovings (see averages in Table 1), respectively. These positive hybrid effects can be seen as a result of the synergistic strengthening of both fibers. In particular, the ultimate stress and strain were increased by about 38 and $25 \%$, respectively, compared with those of the GF roving. The corresponding stiffness after the first peak (CF rupture) was $54.5 \mathrm{MPa}$, about $20 \%$ larger than the elastic modulus (45 MPa) of the GF roving. Tensile stress-strain relations of 2 samples of the hybrid FRP sheets are plotted in Fig. 6, where the LVDT data were used to determine strains, in comparison with that of steel. Again, it is noted that the stress was calculated as the measured load divided by the cross-sectional areas of $A_{H F}$ until the first peak (CF rupture) and $A_{G F}$ after CF rupture.

\subsection{Second Test Program}

In this study, the roving properties of each fiber were not obtained; rather, the impregnated carbon FRP and glass FRP sheets were tested in tension to obtain each fiber's properties. Unfortunately, the results of the impregnated glass FRP sheets were misplaced; thus, the ultimate strain $\left(\varepsilon_{u_{-} G F}=0.0176\right)$ from the roving tests of the first test program is adopted for further analysis, as the fibers used for both programs were manufactured by the same vendor. For the elastic modulus of GF, the average stiffness of the hybrid sheets after CF rupture can be generally taken into account assuming that the Young's modulus $\left(E_{G F}=67.3 \mathrm{GPa}\right)$ is almost the same as that of GF. Finally, the ultimate stress $\left(f_{u_{-} G F}=1,185 \mathrm{MPa}\right)$ is taken as the product of 0.0176 and $67.3 \mathrm{GPa}$.

Figure 7 shows selected results of the impregnated carbon FRP and hybrid FRP sheets, with all results provided in Tables 1, 2 and 3. The stress was taken to be the load divided by the cross-sectional area of the whole fibers until $\mathrm{CF}$ rupture and by the area of the GF only after CF rupture. The cross-sectional area of the sheet was determined based on the number of each fiber roving and its cross-sectional area estimated using the roving weight and Specific Gravity $\left(\rho_{C F}=1.8 ; \rho_{G F}=2.54\right)$, where the weight was measured using a micro-digital scale with an accuracy of

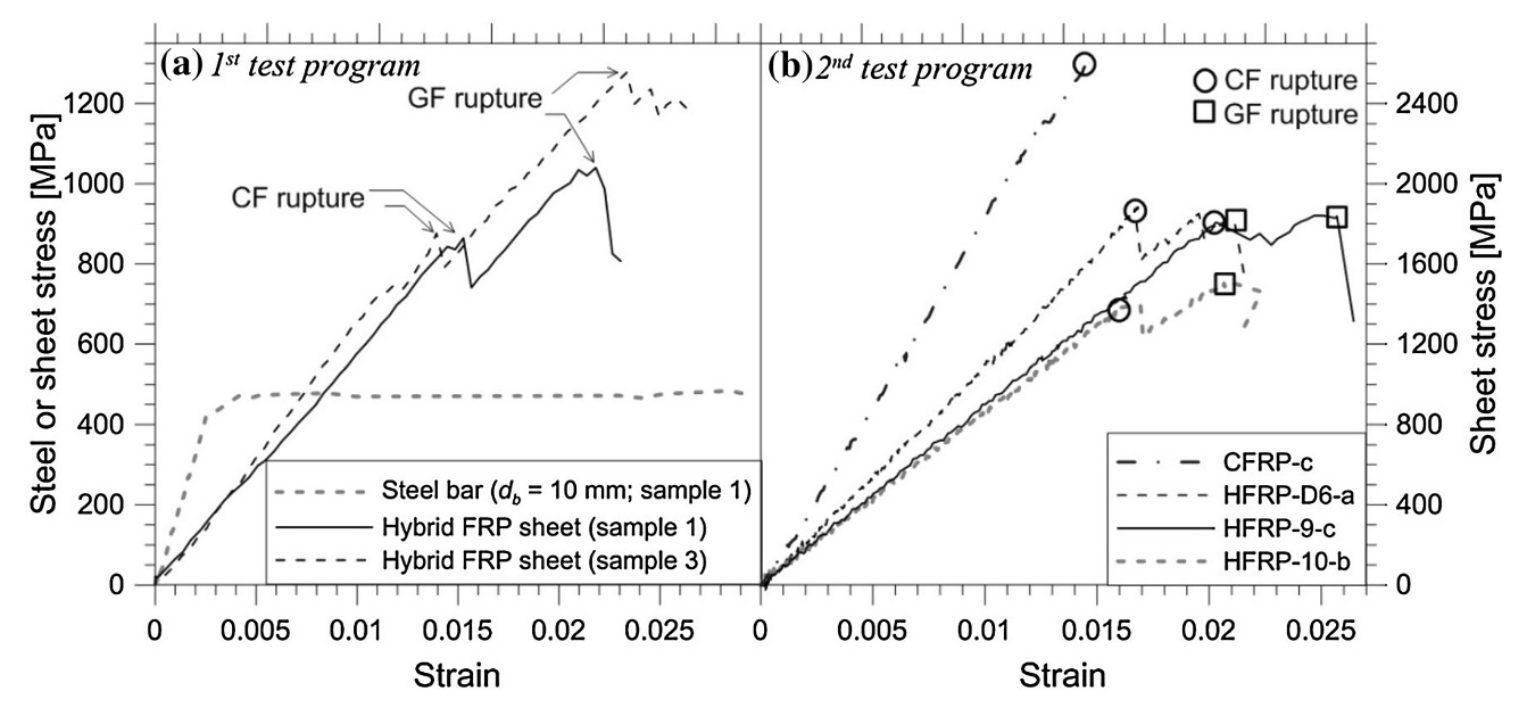

Fig. 6 Measured stress-strain curves of selected carbon FRP and hybrid FRP sheets and conventional steel. 

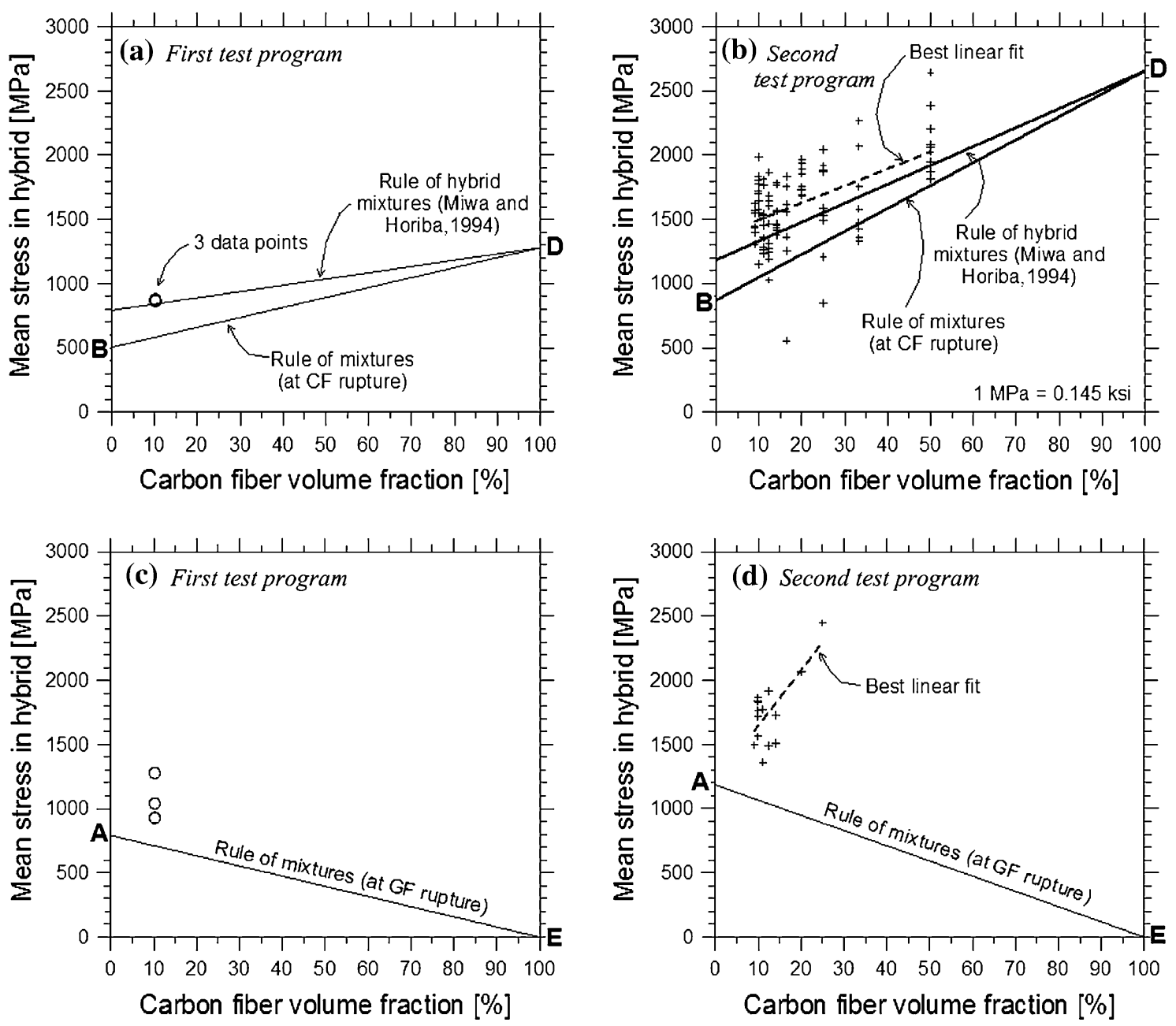

Fig. 7 Identification of hybrid effects.

$\pm 1 \times 10^{-5} \mathrm{~g}$. This does not include the area of the epoxy. In addition, it appears that the type of epoxy resins did not affect the tensile behavior of the sheets much. There was no clear evidence of different performance between the sheets impregnated using the $\mathrm{J}$ type and $\mathrm{K}$ type epoxy resins.

The ultimate stresses (average $f_{u_{C F}}=2,656 \mathrm{MPa}$ ) and elastic moduli (average $E_{C F}=202 \mathrm{MPa}$ ) of the impregnated carbon FRP sheets are lower than those $\left(f_{u_{-} C F}=4,900 \mathrm{MPa}\right.$; $E_{C F}=230 \mathrm{GPa}$ ) of a carbon filament that the manufacturer reported. The differences are much less compared with those between the filament and rovings. The average ultimate strain $\left(\varepsilon_{u_{C} C F}\right)$ of the CF sheets is 0.013 , which is smaller than all but three of 91 ultimate strains (average $\varepsilon_{u} C_{H F}=0.018$ ) of the hybrid sheet coupons at CF rupture. This is evidently due to the hybrid effect. For the hybrid FRP sheets, the ratio of (GF/CF) also affected the overall behavior. As the $(\mathrm{GF} / \mathrm{CF})$ ratio increased, both the strains at $\mathrm{CF}$ and GF ruptures generally increased (Table 3). In the following section, more detailed investigations are conducted in connection with the theoretical models.

\section{Comparison with the Rule of Mixtures}

Experimental results corresponding to line B-D in Fig. 5 are obtained using the measured strains of hybrid FRP coupons at $\mathrm{CF}$ rupture and the measured loads, summarized in Column (4) of Tables 2 and 3, and depicted in Fig. 7a, b. The significantly increased stresses relative to line B-D are noted. The average strain $\left(\varepsilon_{u_{-} C_{-} H F}\right)$ at CF rupture is about $35 \%$ higher than $\varepsilon_{u C F}$. For $(\mathrm{GF} / \mathrm{CF})$ ratios higher than $(4.4 / 1)$, an increase of about $45 \%$ occurred, whereas for $(\mathrm{GF} / \mathrm{CF})$ ratios lower than (4.4/1), the increase was about $22 \%$. In general, the value of $\varepsilon_{u_{-} C_{-} H F}$ increases as the $(\mathrm{GF} / \mathrm{CF})$ ratio increases. This indicates that a constant increase in failure strain, when the hybrid effect is expected, may not be valid (e.g., $50 \%$ or 0.01 strain increase). The difference between the measured stresses of $f_{u_{-} C_{-} H F}$ and the predicted stresses of $f_{u_{-} C_{-} H F}$ based on Eq. (2) is only about $10 \%$ (see Columns (4) and (8) of Tables 2 and 3), indicating that the rule of "hybrid" mixtures suggested by Miwa and Horiba (1994) works better than the rule of mixtures for fiber composites, which underestimates the experimental values by about $35 \%$.

Based on these results, the positive hybrid effect is evident for hybrid FRP sheets that are usually used for repair and retrofit of the concrete structures, and these hybrid effects include the stress and strain of the hybrid FRP sheet at CF rupture (but not Young's modulus). The Young's modulus of the hybrid FRP sheet until CF rupture is almost the same as that of the carbon FRP sheet $(1 \%$ difference on average). 
Similarly, experimental results corresponding to the line A-E in Fig. 5 are examined. The average stress $\left(f_{u_{-} G_{-} H F}=\right.$ $1,094 \mathrm{MPa})$ at $\mathrm{GF}$ rupture was monitored from three specimens of the first test program with the $(\mathrm{GF} / \mathrm{CF})$ ratio of $(8.8 /$ 1). This is about $40 \%$ higher than the corresponding point $\left.\left(f_{u_{G} G F} \times\left(A_{G F} / A_{H F}\right)\right]=771 \mathrm{MPa}\right)$ in Fig. $7 \mathrm{c}$ (see Column (12) of Table 2). The average ultimate strain $\left(\varepsilon_{u_{-} G_{-} H F}=\right.$ $0.022)$ of the hybrid FRP is also higher by $40 \%$ relative to the product ( $=0.0158)$ of $\varepsilon_{u G F}$ and $\left(A_{G F} / A_{H F}\right)$. This is clearly inconsistent with the rule of mixtures. Furthermore, the average increase in stress after $\mathrm{CF}$ rupture divided by the average increase in strain $(54.7 \mathrm{GPa})$ is somewhat higher than the elastic modulus $\left(E_{G F}=45 \mathrm{GPa}\right)$ of the $\mathrm{GF}$ roving (by about $20 \%$ ).

Unlike the first test program, the GF rupture was not captured well for many specimens of the second test program because of the strain gauge or epoxy failure prior to GF rupture, and the disruption of testing once the strain gauge or epoxy failed. Therefore, direct comparisons between $f_{u_{-} G_{-} H F}$ and $\left(f_{u_{-} G F} \mathrm{x}\right.$ $[\mathrm{GF} /(\mathrm{GF}+\mathrm{CF})])$ or between $\varepsilon_{u_{G F}}$ and $\left(\varepsilon_{u_{G} H_{F}} \mathrm{x}[\mathrm{GF} /\right.$ $(\mathrm{GF}+\mathrm{CF})])$ are not possible due to the absence of the GF properties. The averages of the 14 measured values of $f_{u_{-} G \_H F}$ and $\varepsilon_{u G H F}$ are $1,759 \mathrm{MPa}$ and 0.022 , respectively, which are generally higher than the roving test results of the first program. Interestingly, there is a tendency of increasing failure stress $\left(f_{u G H F}\right)$ with decreasing $(\mathrm{GF} / \mathrm{CF})$ ratio or increasing carbon volume fraction (Fig. 7). This means that both the initial stiffness and pseudo-ductility can be obtained even with lower (GF/ $\mathrm{CF}$ ) ratios (see Figs. 6a, 8 and 9). sideration of the hybrid effect rule of mixtures is noteworthy, the data appear not to indicate different ultimate strains depending on the $(\mathrm{GF} / \mathrm{CF})$ ratio (vs ultimate stresses). There is a need for further experimental investigation for the ultimate stress and strain in relation with the $(\mathrm{GF} / \mathrm{CF})$ ratio.

Based on the two independent programs investigated, it can be concluded that the hybrid effects are positive in terms of the ultimate stress and strain at GF rupture for all (GF/CF) ratios. Note that these positive effects are contrary to the

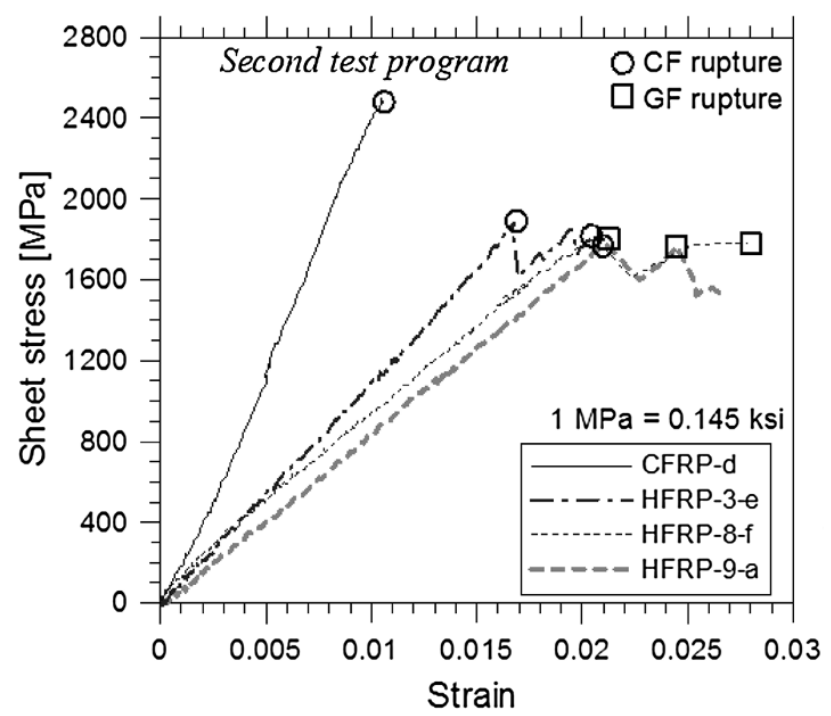

Fig. 8 Pseudo-ductility of hybrid FRP sheets noted from measured stress-strain curves. prediction made by Pan and Postle (1996) for micro-fibers embedded in the matrix. This seems to be related to the degree of coupling between two different fibers. The use of hybrid FRP sheets consisting of CF and GF rovings has been proven to be a very effective means to promote synergistic hybrid effects. In a similar manner, the hybrid effect of uniaxial hybrid FRP sheets that are made of three different fiber rovings could be investigated.

Figure $6 \mathrm{a}$ shows the stress-strain relationship for the two coupons tested in the first test program, where pseudo-ductility was observed. This is consistent with the rule of mixtures which suggests the recovery of the stress after $\mathrm{CF}$ rupture when a point representing the $(\mathrm{GF} / \mathrm{CF})$ ratio is located to the left of Point $\mathrm{C}$ in Fig. 5. On the other hand, the data reported from the second test program exhibit a high degree of ductility after $\mathrm{CF}$ rupture even for low (GF/CF) ratios (Figs. $6 \mathrm{~b}$ and 8 ). Because the data are quite limited, additional tests would be helpful to evaluate the stress recovery after CF rupture.

\section{Proposed Stress-Strain Relationship for Hybrid FRP Sheets}

When the moment and shear capacities of concrete members strengthened with hybrid FRP sheets are determined, a stress-strain or force-strain relationship of the hybrid FRP sheets would be needed. The stress-strain relationship of Eqs. (3) and (4), which is based on the rule of mixtures, does not account for the identified positive hybrid effects. In this study, based on the review on the test results, the following stress-strain relationship for hybrid carbonglass hybrid FRP sheets is proposed.

$$
\begin{aligned}
& \text { If } \varepsilon_{H F} \leq \varepsilon_{u_{-} C} \_H F \\
& f_{H F}=E_{H F} \varepsilon_{H F}=\left[E_{C F}\left(\frac{A_{C F}}{A_{H F}}\right)+E_{G F}\left(\frac{A_{G F}}{A_{H F}}\right)\right] \varepsilon_{H F} \\
& \text { If } \quad \varepsilon_{u \_C \_H F}<\varepsilon_{H F} \leq \varepsilon_{u \_G \_H F} \quad f_{H F}=E_{G F} \varepsilon_{H F}
\end{aligned}
$$

where $\varepsilon_{u_{-} C_{-} H F}$ is taken as $\left(f_{u_{-} C_{-} H F} / E_{H F}\right) ; f_{u_{-} C_{-} H F}$ can be estimated using Eq. (2); and $\varepsilon_{u_{-} G_{-} H F}$ is the strain of hybrid FRP sheets at GF rupture, suggested to be $\sim 0.022$ when a (GF/CF) ratio is larger than (4/1), respectively. The suggested values are based on the assessed data from the two test programs. It is noted that the elastic moduli in the proposed stress-strain relationship do not reflect the positive hybrid effect since the increased properties are minor, and that after $\mathrm{CF}$ rupture the stress of $f_{H F}$ should be paired with $A_{G F}$ (not $A_{H F}$ ) to determine the force, assuming that $\mathrm{CF}$ no longer resists any tension.

The analytical stress-strain relationships with and without consideration of hybrid effects are depicted in Fig. 9 for a variety of $(\mathrm{GF} / \mathrm{CF})$ ratios. The former is expressed in the form of Eqs. (5) and (6), while the latter is expressed in the form of Eqs. (3) and (4). It is noted that $\varepsilon_{u} C_{H F}$ is a function of the $(\mathrm{GF} / \mathrm{CF})$ ratio, whereas a fixed value of $\varepsilon_{u_{-} G_{-} H F}$ of 0.022 is proposed. Additionally, strains of $\varepsilon_{u_{-} C F}$ and $\varepsilon_{u_{-} G F}$ are suggested to be 0.013 and 0.018 , respectively, and elastic moduli of $E_{C F}$ and $E_{G F}$ to be 202 and $67 \mathrm{GPa}$. If the rule of mixtures is 

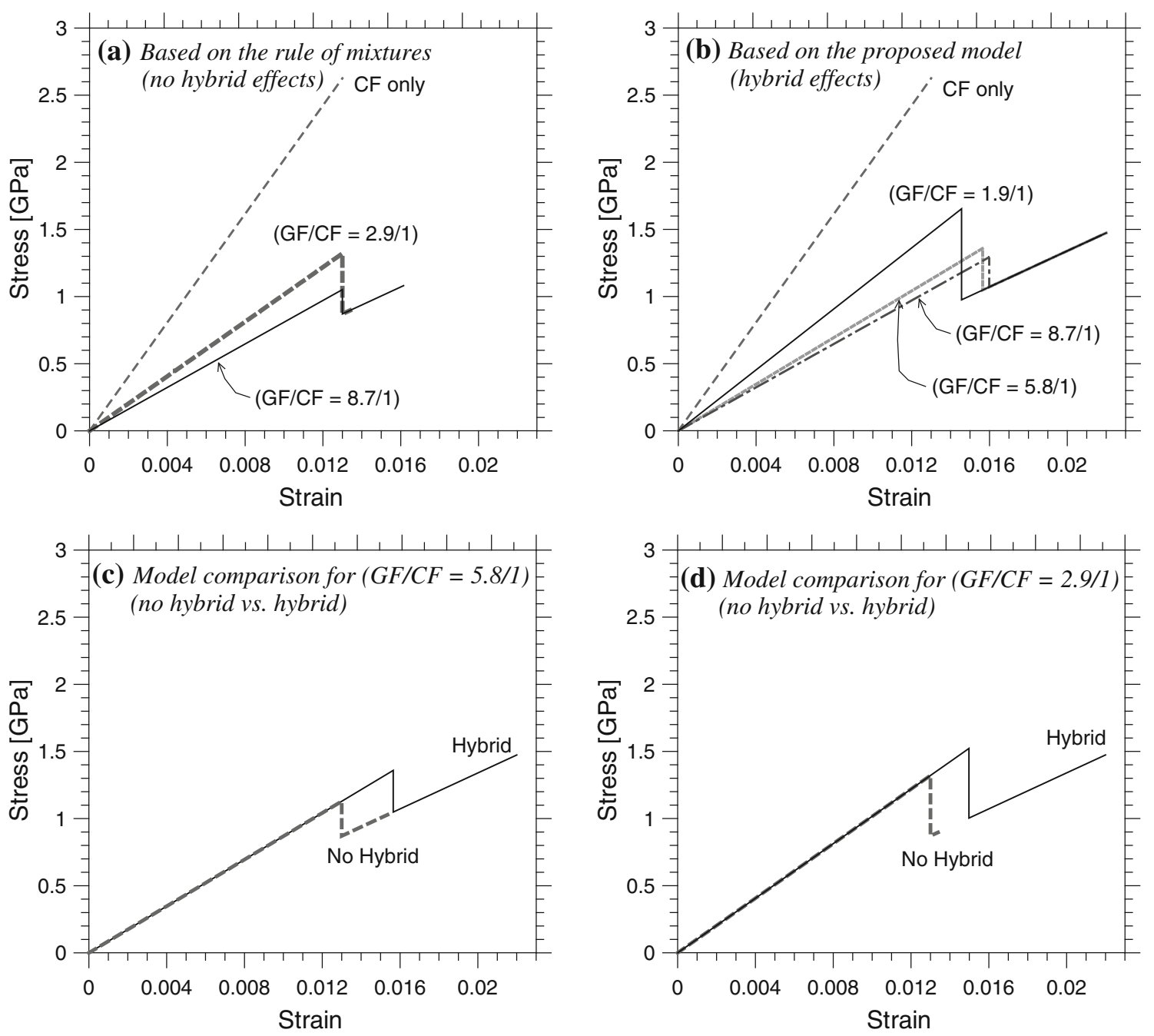

Fig. 9 Models for stress-strain relationship of hybrid FRP sheets with various (GF/CF) ratios.

applied, pseudo-ductility can only be achieved when a (GF/ $\mathrm{CF}$ ) ratio is not less than (8.8/1) (Fig. 9a). If the hybrid effect is considered, which has been demonstrated in this study, pseudo-ductility is characterized in almost all (GF/CF) ratios (Fig. 9b). Furthermore, a strain at $\mathrm{CF}$ rupture is even higher than that at the second fiber (GF) rupture without the consideration of the hybrid effect (Fig. 9c, d). This is one of the greatest advantages that hybridized carbon and GF can offer. Regarding the effect of the $(\mathrm{GF} / \mathrm{CF})$ ratio, the hybrid model shows better performance in terms of the stiffness for lower (GF/CF) ratios, and the same ductility for all (GF/CF) ratios, still substantially higher than that of CF or GF. Such behaviors are seen in Figs. $6 \mathrm{a}$ and 8. However, a high (GF/CF) ratio (e.g., 8.7/1) is recommended because the reduction of the cross-sectional area of the FRP sheet with a very low $(\mathrm{GF} / \mathrm{CF})$ is drastic and cost effectiveness is at its maximum when the highest $(\mathrm{GF} / \mathrm{CF})$ ratio is used.

\section{Verification of Hybrid Effects of FRP in Combination with Concrete Beams}

The writers of this paper previously tested plain concrete beams and reinforced concrete (RC) beams with hybrid FRP sheets or carbon FRP sheets (Choi et al. 2011). Figure 10 compares load-displacement relations for the tested plain concrete beams. All beams failed in a relatively brittle manner due to the fiber rupture. It is notable that the $\mathrm{CF}$ ruptures in concrete beams with hybrid FRP sheets [H-3-7/ 1(a) and H-3-7/1(b)] occurred at larger displacements than CF ruptures in concrete beams with carbon FRP sheets only [C-3(a) and C-3(b)], respectively. This clearly verifies positive hybrid effect in terms of $\mathrm{CF}$ rupture. Moreover, positive hybrid effect for GF rupture was observed in that GF ruptures in beams with hybrid FRP sheets [H-3-7/1(a) and H-3-7/1(b)] occurred at slightly to substantially larger displacements than GF ruptures in beams with glass FRP sheets only [G-3(a) and G-3(b)], respectively. Figure 11 depicts load versus mid-span deflection relations for $\mathrm{RC}$ beams strengthened with hybrid FRP sheets or carbon FRP sheets. The hybrid FRPstrengthened $\mathrm{RC}$ beams underwent both $\mathrm{CF}$ and GF ruptures at more than 26 and $30 \mathrm{~mm}$ displacements, respectively, whereas the carbon FRP-strengthened RC beam began to fail at a much lower displacement of $\sim 24 \mathrm{~mm}$. As such, the positive hybrid effect in terms of CF rupture was also verified for hybrid FRP sheets bonded to RC beams. Such a positive hybrid effect was consistently found regardless of the number of hybrid FRP sheet layers (1 ply to 3 plies). 


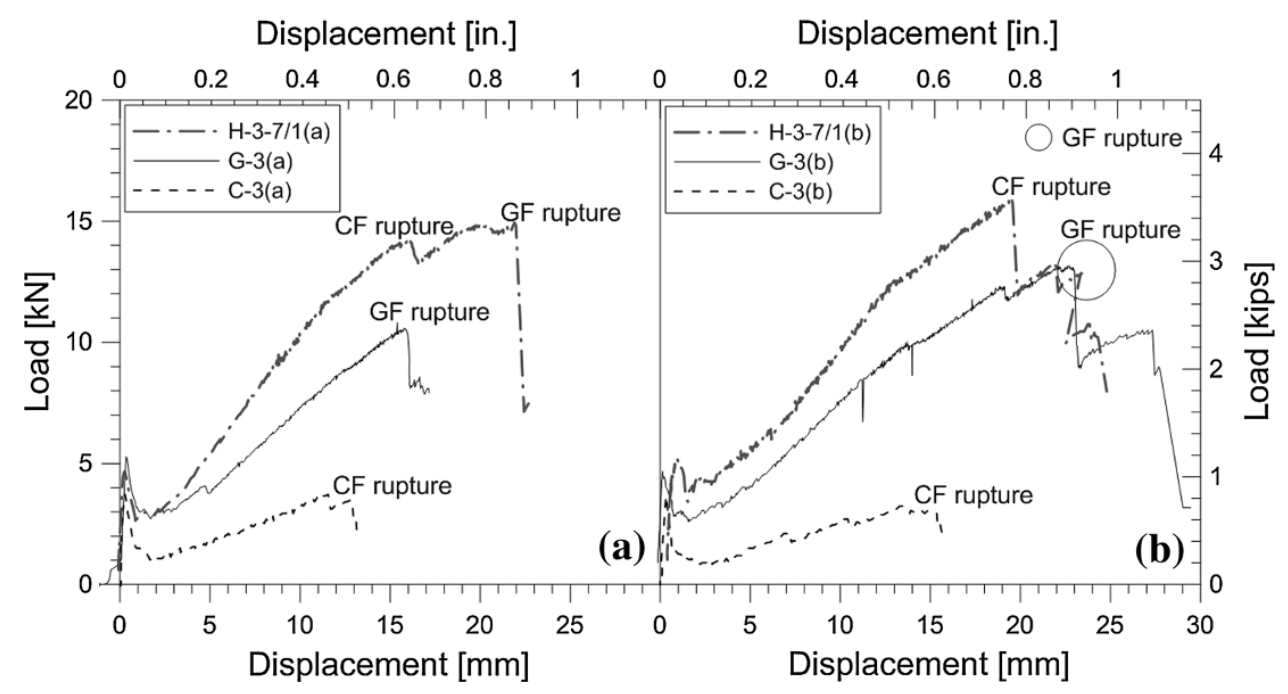

Fig. 10 Measured load-versus-midspan deflection relations for plain concrete beams (reproduced from Choi et al. 2011).

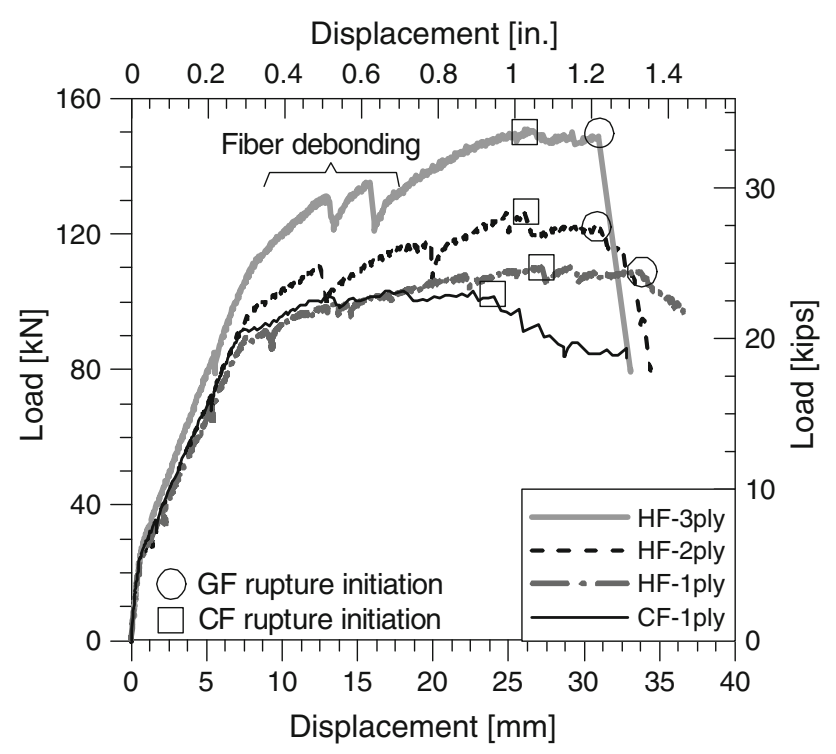

Fig. 11 Measured load-versus-midspan deflection relations for RC beams (reproduced from Choi et al. 2011).

\section{Conclusions}

The purposes of the use of uniaxial hybrid FRP sheets in the repair of existing concrete structures can be to achieve pseudo-ductility and utilize their hybrid effects. In this study, the tensile test results from a total of 94 hybrid carbon-glass FRP sheets and 47 carbon and GF rovings or sheets were investigated in depth. Based on the investigation, a number of conclusions are made as follows:

(1) The three types of grips used for the roving tests are effective. In particular, the grip with $90^{\circ}$ sandwich laminates using the same fiber rovings was very sound.

(2) The two epoxy resins ( $\mathrm{J}$ and $\mathrm{K}$ types) sustained strains up to about 0.02 and 0.03 , respectively; however, an ultimate strain of about 0.04 is recommended to prevent epoxy failure prior to fiber rupture. Neither type of the epoxy resins affected the tensile behavior of the sheets much.
(3) The elastic moduli of hybrid FRP sheets generally correspond to the rule of mixtures.

(4) The strains at CF and GF ruptures of the hybrid sheets are about 0.018 and 0.022 , respectively, on average, which are substantially higher than the ultimate strains of each CF and GF (0.013 and 0.018). A trend of increased strain at $\mathrm{CF}$ rupture for increased $(\mathrm{GF} / \mathrm{CF})$ ratio was observed, while there is no clear indication of different strains at GF rupture depending on the $(\mathrm{GF} /$ CF) ratio.

(5) The stresses at CF rupture of the hybrid sheets are significantly higher than those predicted based on the rule of mixtures, differing by about $40 \%$, but are quite close to those predicted based on the rule of hybrid mixtures developed by Miwa and Horiba (1994) (within about $10 \%$ difference). Additionally, the stresses at GF rupture are considerably higher than those predicted based on the rule of mixtures, by about $80 \%$. The discovery of these positive hybrid effects is a significant advance.

(6) A general trend of increased stress at GF rupture for decreased $(\mathrm{GF} / \mathrm{CF})$ ratio was observed. This signals that both the initial stiffness and pseudo-ductility could be obtained even with a very low $(\mathrm{GF} / \mathrm{CF})$ ratio. However, given the limited data, additional research would be needed to verify this trend.

(7) The identified hybrid effects are evident for all (GF/CF) ratios. When carbon and GF are hybridized, an ultimate strain at the first fiber (carbon) rupture could be even higher than the ultimate strain of the second fiber (glass only).

(8) The positive hybrid effects at both CF and GF ruptures might be shown only in the hybrid FRP sheets that are made of CF and GF rovings. If each fiber had been mixed in a roving, the positive hybrid effects might have not been found.

(9) The positive hybrid effects at both CF and GF ruptures were clearly verified by the structural member-level tests of both plain concrete beams and RC beams. 


\section{Acknowledgments}

The research was supported by the National Research Foundation of Korea (NRF) Grants (Nos. 2005042103561 and 2012005905) and U.S. DOT Grant (No. DTRT06-G0016/OTCREOS10.1-21). Additionally, the authors would like to acknowledge Dr. Chin-Yong Lee of CareCon Co. for his generous donation of materials and technical supports. The opinions, findings and conclusions in this paper are those of the authors and do not necessarily represent those of the sponsors.

\section{Open Access}

This article is distributed under the terms of the Creative Commons Attribution License which permits any use, distribution, and reproduction in any medium, provided the original author(s) and the source are credited.

\section{References}

ACI Committee. (1998). 503, Use of Epoxy Compounds with Concrete (ACI 503R-93; Reapproved 1998), ACI, Farmington Hills, Mich., p. 28.

ACI Committee. (2002). 440, Guide for the Design and Construction of Externally Bonded FRP Systems for Strengthening Concrete Structures (ACI 440.2R-02), ACI, Farmington Hills, Mich., p. 45.

ASTM International. (2008). ASTM D638-08 Standard Test Method for Tensile Properties of Plastics, West Conshohocken, Penn: ASTM International, p. 16.

ASTM International. (2008). ASTM D3039/D3039M-08 Standard Test Method for Tensile Properties of Polymer Matrix Composite Materials, ASTM International, West Conshohocken, Pennsylvania, p. 13.

Aveston, J., \& Sillwood, J. M. (1976). Synergistic fibre strengthening in hybrid composites. Journal of Materials Science, 11(10), 1877-1883.

Bunsell, A. R., \& Harris, B. (1974). Hybrid carbon and glass fibre composites. Composites, 5, 157-164.
CAN/CSA. (2007). S806-02 (R2007), Design and construction of building components with fibre-reinforced polymers, CSA, Mississauga, Canada, p. 206.

Choi, D.-U., Kang, T. H.-K., Ha, S-. S., Kim, K.-H., \& Kim, W. (2011). Flexural and bond behavior of concrete beams strengthened with hybrid carbon-glass fiber-reinforced polymer sheets. ACI Structural Journal, 108(1), 90-98.

Chou, T.-W., \& Kelly, A. (1980). Mechanical properties of composites. Annual Reviews of Material Science, 10, 229-259.

Cox, H. L. (1952). The elasticity and strength of paper and other fibrous materials. British Journal of Applied Physics, 3, $72-79$.

Grace, N. F., Abel-Sayed, G., \& Ragheb, W. F. (2002). Strengthening of concrete beams using innovative ductile fiber-reinforced polymer fabric. ACI Structural Journal, 99(5), 692-700.

Harris, H. G., Somboonsong, W., \& Frank, K. K. (1998). New ductile hybrid FRP reinforcing bar for concrete structures. ASCE Journal of Composites for Construction, 2(4), 28-36.

Manders, P. W., \& Bader, M. G. (1981). The strength of hybrid glass/carbon fibre composites: Part 1. Failure strain enhancement and failure mode. Journal of Materials Science, 16, 2233-2245.

Marom, G., Fischer, S., Tuler, F. R., \& Wagner, H. D. (1978). Hybrid effects in composites: Conditions for positive or negative effects versus rule of mixtures. Journal of Materials Science, 13, 1419-1426.

Miwa, M., \& Horiba, N. (1994). Effects of fibre length on tensile strength of carbon/glass fibre hybrid composites. Journal of Materials Science, 29, 973-997.

Nanni, A., Henneke, M. J., \& Okamoto, T. (1994). Tensile properties of hybrid rods for concrete reinforcement. Construction and Building Materials, 8(1), 27-34.

Pan, N., \& Postle, R. (1996). Tensile strengths and the hybrid effects of hybrid fibre composites: A probabilistic approach. Transitions of the Royal Society in London, Series A, 354, 1875-1897.

Phillips, L. N. (1976). The hybrid effect-does it exist? Composites, 7, 7-8. 